\title{
NEUTRAL HYDROGEN (21 CENTIMETER) ABSORPTION IN SEYFERT GALAXIES: EVIDENCE FOR FREE-FREE ABSORPTION AND SUBKILOPARSEC GASEOUS DISKS
}

\author{
J. F. GALLIMORE \\ National Radio Astronomy Observatory, ${ }^{1} 520$ Edgemont Road, Charlottesville, VA 22903; jgallimo@nrao.edu \\ S. A. BAUM AND C. P. O’DeA \\ Space Telescope Science Institute, 3700 San Martin Drive, Baltimore, Maryland 21218 \\ A. Pedlar \\ Nuffield Radio Astronomy Laboratories, Jodrell Bank, Macclesfield, Cheshire SK11 9DL, UK \\ AND \\ E. BRINKS \\ Departamento de Astronomía, Universidad de Guanajuato, Apdo. Postal 144, Guanajuato, C.P. 36000, Mexico \\ Received 1999 March 16; accepted 1999 June 5
}

\begin{abstract}
Active galaxies are thought to be both fueled and obscured by neutral gas removed from the host galaxy and funneled into a central accretion disk. We performed a VLA imaging survey of $21 \mathrm{~cm}$ absorption in Seyfert and starburst nuclei to study the neutral gas in the near-nuclear environment. With the exception of NGC 4151, the absorbing gas traces $100 \mathrm{pc}$-scale, rotating disks aligned with the outer galaxy disk. These disks appear to be rich in atomic gas relative to nuclear disks in nonactive spirals. We find no strong evidence for rapid infall or outflow of neutral hydrogen, but our limits on the mass infall rates are compatible with that required to feed a Seyfert nucleus. Among the galaxies surveyed here, neutral hydrogen absorption traces parsec-scale gas only in NGC 4151. Based on the kinematics of the absorption line, the disk symmetry axis appears to align with the radio jet axis rather than the outer galaxy axis. The most surprising result is that we detect no $21 \mathrm{~cm}$ absorption toward the central radio sources of the hidden Seyfert 1 nuclei Mrk 3, Mrk 348, and NGC 1068. Moreover, $21 \mathrm{~cm}$ absorption is commonly observed toward extended radio jet structure but appears to avoid central, compact radio sources in Seyfert nuclei. To explain these results, we propose that $21 \mathrm{~cm}$ absorption toward the nucleus is suppressed by either free-free absorption, excitation effects (i.e., enhanced spin temperature), or rapid motion in the obscuring gas. Ironically, the implications of these effects is that the obscuring disks must be small, typically not larger than a few tens of parsecs.
\end{abstract}

Subject headings: galaxies: active — galaxies: ISM — galaxies: Seyfert — galaxies: starburst galaxies: structure - radio lines: galaxies

\section{INTRODUCTION}

The commonly accepted model explaining the power source for active galactic nuclei (AGNs) is a massive black hole fed by an accretion disk. The advantage of this model is the high efficiency of conversion of gravitational energy to radiant energy, but the main difficulty is the removal of angular momentum. Unlike stars, which constitute a dissipationless medium, gas and dust can lose orbital energy and angular momentum through collisions and shocks. At least in Seyfert galaxies, but probably in all AGNs, the host galaxy reserves a mass of neutral ISM comparable to or exceeding the lifetime needs of the AGN. Except in galaxy clusters, the mass of the hot phase component of the ISM is insufficient to fuel the most luminous AGNs over their duration. Although the transport mechanism remains a mystery, the conventional model holds that AGNs are powered by neutral ISM removed from the host galaxy (e.g., Rees 1984).

Important variants on the standard model are the unifying schemes for AGNs. The dust associated with the neutral ISM can obscure a range of sight lines to the AGN, affecting its outward appearance. It is proposed that both

\footnotetext{
${ }^{1}$ The National Radio Astronomy Observatory is operated by Associated Universities, Inc., under contract with the National Science Foundation.
}

broad-line (e.g., Seyfert 1) and narrow-line (Seyfert 2) AGNs are identical, but the broad-line region (BLR) of Seyfert 2 galaxies may be obscured by a dusty ring, viewed more nearly edge-on, surrounding the active nucleus (this model is reviewed in, e.g., Lawrence 1987; Antonucci 1993; Ward 1996). There are a handful of hidden Seyfert 1 galaxies discovered by spectropolarimetry (e.g., Antonucci \& Miller 1985; Miller \& Goodrich 1990; Tran, Miller, \& Kay 1992), but it is not clear whether the model is generally applicable. The detection of relatively high X-ray-obscuring columns toward Seyfert 2 nuclei warrants the question of the nature and scale of the obscuring medium (e.g., Awaki et al. 1991; Mulchaey, Mushotzky, \& Weaver 1992; Mulchaey et al. 1993; Smith \& Done 1996; Turner et al. 1997).

We have observed a sample of 13 radio-bright Seyfert galaxies in $\mathrm{H} \mathrm{I}(21 \mathrm{~cm})$ absorption to explore the distribution and kinematics of the neutral ISM in the vicinity of the AGN. There are two primary advantages of this technique. First, absorption lines are potentially more sensitive than emission lines, the former limited primarily by the brightness of the background source rather than the column density or emission measure of the gas. Second, one can use powerful aperture-synthesis arrays to resolve the absorption-line gas on scales as small as the background radio source (i.e., potentially down to subparsec scales). Conversely, although the absorption experiment may not resolve the background source, the structure of the back- 
ground source may be known from independent continuum studies, and so the effective resolution of the absorption experiment is improved. A third advantage is that one knows for certain that the absorption-line gas must lie in front of the background source, removing the ambiguity suffered by emission-line studies. Of course, the primary limitation is that one can detect and resolve absorption-line gas only to the extent of the background source. In addition, because the opacity is inversely proportional to the excitation temperature, absorption lines are weighted more heavily by colder clouds.

We present this work as follows. Section 2 describes the sample selection, observations, and data reduction techniques. Section 3 presents the data and detection statistics. In $\S 4$ we discuss the properties of the $\mathrm{H}$ I absorbing gas, including quantitative treatments of the spin temperatures and $\mathrm{H}$ I column densities. We also compare the observed $\mathrm{H}$ I columns with hydrogen columns based on X-ray spectroscopy. Section 5 describes the distribution and kinematics of the $\mathrm{H}$ I absorption based on the application of rotating disk models. Sections 6 and 7 discuss the implications of these observations on the standard model for AGNs, and we summarize our main conclusions in $\S 8$.

\section{OBSERVATIONS AND DATA REDUCTION}

\subsection{Sample Selection}

The selection criteria for this survey were spiral galaxies with evidence for nuclear activity and radio brightness $S_{v}>50 \mathrm{mJy}$ as resolved by the VLA A-Array. In essence this survey is a radio flux-limited sample comprising mainly Seyfert galaxy nuclei. Our original survey consisted of 24 active spirals, but, owing to a technical problem with our first observation (VLA program AB 605 on 1991 July 19), we were unable to recover the $\mathrm{H}$ i spectra for these sources. We received a reduced time allocation for a repeat observation on 1992 November 22. Time and local sidereal time (LST) constraints reduced our sample to a subset of 13 of the brightest sources (VLA program AB 658).

\subsection{Observations}

We observed the sample galaxies with the VLA operating in its A-configuration, i.e., the longest baseline configu- ration, with baselines ranging from $\sim 3.2-170 \mathrm{k} \lambda$ at $21 \mathrm{~cm}$. The spatial resolutions in the image plane are typically $\sim 1$."5-2". A summary of the main observing parameters are provided in Table 1. Receivers were tuned to the appropriately redshifted wavelength of the $\mathrm{H}$ I $21 \mathrm{~cm}$ line (rest frequency $v=1420.406 \mathrm{MHz}$ ). We used the optical definition for velocity and referenced the velocities to the heliocentric frame. Eleven sources were observed in 4-IF mode, with paired intermediate frequencies (IFs) tuned redward and blueward of the systemic velocity to expand the velocity range covered by the receivers. The bandwidths are 3.1 $\mathrm{MHz}$ divided into 32 channels. In velocity units, the channel widths are $\sim 21 \mathrm{~km} \mathrm{~s}^{-1}$, and, accounting for channel overlaps between the IF pairs, the total bandwidth is $\sim 1050 \mathrm{~km}$ $\mathrm{s}^{-1}$. The two remaining sources (NGC 2992 and NGC 5506) were observed using $1 \mathrm{~A}$ mode covering a single bandpass of $6.25 \mathrm{MHz}$, again with channel widths of $\sim 21$ $\mathrm{km} \mathrm{s}^{-1}$.

Source observations interleaved with shorter scans of a point-source calibrator for phase referencing. Each source observation was accompanied by a scan of a bright flux standard, either 3C 48 or 3C 286 . We also used these sources for bandpass calibration to remove the residual channel-to-channel complex gain variations.

\subsection{Data Reduction}

We reduced the data using standard procedures in AIPS. The flux scale was set by scans of the radio-continuum standards 3C 48 and 3C 286, defined according to Baars et al. (1977). Absolute calibration of interferometric phase was determined by point-source models for the phase reference calibrators. We then employed 2-3 iterations of selfcalibration on each of our targets, properly including all of the confusing sources brighter than $5 \mathrm{mJy}$ within two primary beam widths. The final CLEAN-deconvolved models were subtracted from the $(u, v)$ database. We generated spectral-line cubes for each target by Fourier transform. The cubes were inspected for residual continuum emission or bandpass gradient not subtracted by the CLEAN-component model. For stronger sources, we subtracted a linear fit to the spectral baseline, but for weaker sources it sufficed to remove a continuum mean. All

TABLE 1

OBSERVING PARAMETERS

\begin{tabular}{|c|c|c|c|c|c|}
\hline \multirow[b]{2}{*}{ SOURCE } & \multirow{2}{*}{$\begin{array}{c}t_{\text {int }} \\
\text { (minutes) }\end{array}$} & \multirow{2}{*}{$\begin{array}{c}\mathrm{rms} \\
\left(\mathrm{mJy} \mathrm{beam}^{-1}\right)\end{array}$} & \multirow{2}{*}{$\begin{array}{l}\text { CONTINUUM } \\
\left(\mathrm{mJy} \mathrm{beam}^{-1}\right)\end{array}$} & \multicolumn{2}{|c|}{ BEAM } \\
\hline & & & & $(\operatorname{arcsec})$ & (deg) \\
\hline Mrk $3 \ldots \ldots \ldots$. & 20 & 1.5 & 895 & $2.3 \times 1.6$ & 72 \\
\hline Mrk $6 \ldots \ldots \ldots$ & 52 & 1.0 & 176 & $2.1 \times 1.6$ & -14 \\
\hline Mrk $231 \ldots \ldots \ldots$ & 25 & 1.0 & 228 & $1.8 \times 1.6$ & 9 \\
\hline Mrk $348 \ldots . . .$. & 24 & 1.3 & 287 & $1.8 \times 1.5$ & 47 \\
\hline Mrk $668 \ldots \ldots$. & 15 & 2.0 & 736 & $1.9 \times 1.7$ & -53 \\
\hline NGC $1068 \ldots . .$. & 100 & 0.9 & 1395 & $2.8 \times 1.6$ & -23 \\
\hline NGC $2110 \ldots . .$. & 114 & 0.9 & 126 & $2.7 \times 1.5$ & -35 \\
\hline NGC $2639 . . .$. & 60 & 0.8 & 85 & $1.8 \times 1.7$ & 68 \\
\hline NGC $2992 \ldots . .$. & 90 & 0.7 & 49 & $3.7 \times 1.5$ & 17 \\
\hline NGC $3079 . . . .$. & 141 & 0.6 & 83 & $1.7 \times 1.6$ & 21 \\
\hline NGC $3504 \ldots . .$. & 29 & 1.4 & 52 & $1.8 \times 1.7$ & 75 \\
\hline NGC $4151 \ldots . .$. & 49 & 0.8 & 193 & $1.9 \times 1.8$ & 70 \\
\hline NGC $5506 \ldots . .$. & 89 & 0.7 & 223 & $2.0 \times 1.5$ & 9 \\
\hline
\end{tabular}

NoTE.-The reported continuum measurement is the peak flux density of the continuum image but not necessarily the integrated continuum emission from the source. 
residual subtractions were performed in the image (i.e., not $u, v$ ) plane.

A possible source of error in this experiment is a bandpass calibration artifact resulting from temperature variations, and hence length variations, of the waveguide system at the VLA (Carilli 1991; Lilie 1994). The signature is a sinusoidal ripple with a period of $3 \mathrm{MHz}$, matching the bandwidth used in this experiment. Among the sources observed for this experiment, only the nuclear spectrum of NGC 1068 displayed broad spectral features that could be explained by the $3 \mathrm{MHz}$ ripple. To test for this artifact, we generated a spectrum of the phase calibrator for NGC 1068. The phase calibrator showed similar spectral structure, corresponding to a spectral dynamic range of $\sim 400$. We therefore have little confidence in the broad spectral features of the nuclear $21 \mathrm{~cm}$ absorption profile of NGC 1068 .

\section{RESULTS}

We detect $\mathrm{H}$ I absorption toward nine of the 13 sources observed in this survey, making a detection rate of $69 \%$. The breakdown by nuclear activity is three of four Seyfert 1 nuclei detected (Fig. 1; also see Table 2); four of six Seyfert 2 nuclei detected (Fig. 2); and two of three starburst/LINER nuclei detected (Fig. 3). Explicitly, the nondetections are Mrk 668 (Seyfert 1), Mrk 3, and Mrk 348 (both Seyfert 2 with hidden Seyfert 1 nuclei), and NGC 2639 (LINER). We summarize the basic properties of the integrated $\mathrm{H}$ I absorption in Table 3. Parameters of the integrated absorptionline profiles are provided in Table 4.

In several sources, the radio continuum was sufficiently resolved that we could isolate or map the $\mathrm{H}$ I absorption distribution against the extended continuum (see Figs. 4-11). Sources in which the $\mathrm{H} \mathrm{I}$ absorption is unresolved but the radio continuum is resolved are NGC 2110 (Fig. 6), NGC 2992 (Fig. 7), NGC 4151 (Fig. 10), and Mrk 6 (Fig. 11). The continuum and $\mathrm{H}$ I absorption in NGC 1068 are

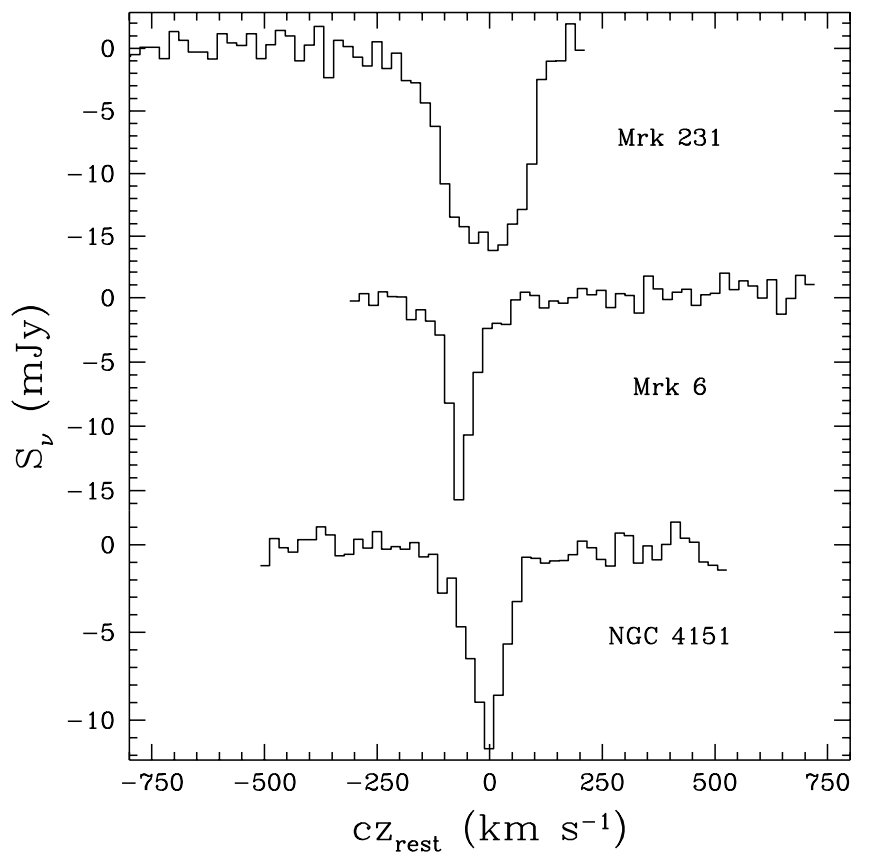

FIG. 1. - Global (spatially integrated) H I absorption spectra of Seyfert 1 nuclei. The spectra are continuum subtracted and corrected back to zero rest velocity based on the observed recessional velocities in Table 2 . The only nondetection among the Seyfert 1 nuclei was Mrk 668.

resolved (Figs. 4 and 5), and the $\mathrm{H} \mathrm{I}$ absorption in NGC 3079 is marginally resolved against the background continuum (Figs. 8 and 9). Follow-up MERLIN observations have better resolved the $\mathrm{H}$ I absorption toward NGC 3079 (Pedlar et al. 1996), NGC 4151 (Mundell et al. 1995), and Mrk 6 (Gallimore et al. 1998). H I absorption toward Mrk 231 has been resolved by the VLBA (Carilli, Wrobel, \&

TABLE 2

SOURCE PROPERTIES

\begin{tabular}{|c|c|c|c|c|c|c|}
\hline Source & $\begin{array}{l}\text { Right Ascension } \\
\text { (1950) }\end{array}$ & $\begin{array}{l}\text { Declination } \\
\quad(1950)\end{array}$ & $\begin{array}{c}c z \\
\left(\mathrm{~km} \mathrm{~s}^{-1}\right)\end{array}$ & Reference & Activity & $\begin{array}{l}\text { Morphology } \\
(\text { NED })^{\mathrm{a}}\end{array}$ \\
\hline Mrk $348 \ldots \ldots \ldots$ & 004604.85 & +314104.5 & $4507 \pm 4$ & 1 & $2(1)$ & $\mathrm{SA}(\mathrm{s}) 0 / \mathrm{a}$ \\
\hline NGC $1068 \ldots \ldots$ & $0240 \quad 07.07$ & -001331.2 & $1150 \pm 5$ & 2 & $2(1)$ & $\mathrm{SA}(\mathrm{rs}) \mathrm{b}$ \\
\hline NGC $2110 \ldots . .$. & 054946.38 & -072800.9 & $2335 \pm 20$ & 3 & 2 & $\mathrm{SAB} 0-$ \\
\hline Mrk $3 \ldots \ldots \ldots . .$. & 060948.24 & +710310.5 & $3998 \pm 6$ & 1 & $2(1)$ & S0 \\
\hline Mrk $6 \ldots \ldots \ldots \ldots$ & 064543.96 & +742909.9 & $5640 \pm 10$ & 4 & 1.5 & $\mathrm{SAB} 0+$ \\
\hline NGC $2639 . . . .$. & $08 \quad 4003.12$ & +502310.2 & $3336 \pm 11$ & 1 & $\mathrm{~L}$ & SA(r)a \\
\hline NGC $2992 \ldots . .$. & $0943 \quad 17.62$ & -140542.9 & $2314 \pm 6$ & 1 & $\mathrm{X}$ & Sa pec \\
\hline NGC $3079 . . . .$. & 095835.01 & +555515.5 & $1124 \pm 10$ & 5 & $\mathrm{~L}$ & $\mathrm{SB}(\mathrm{s}) \mathrm{c}$ \\
\hline NGC $3504 \ldots . .$. & 110028.53 & +281431.3 & $1534 \pm 2$ & 6 & SB & $\mathrm{SAB}(\mathrm{s}) \mathrm{ab}$ \\
\hline NGC $4151 \ldots . .$. & 120801.01 & +394102.1 & $997 \pm 3$ & 7 & 1.5 & $\mathrm{SAB}(\mathrm{rs}) \mathrm{ab}$ \\
\hline Mrk $231 \ldots \ldots \ldots$ & 125405.03 & +570838.2 & $12650 \pm 20$ & 8 & 1 & $\mathrm{SA}(\mathrm{rs}) \mathrm{c} ?$ \\
\hline Mrk $668 \ldots \ldots . .$. & 140445.61 & +284129.3 & $22957 \pm 40$ & 9 & 1 & $\ldots$ \\
\hline NGC $5506 \ldots . .$. & 141039.14 & -025827.1 & $1815 \pm 9$ & 1 & 2 & Sa pec \\
\hline
\end{tabular}

NoTES.-Properties of the sources observed in this experiment. Coordinates mark the $21 \mathrm{~cm}$ continuum peak, not necessarily the nucleus, at $\sim 2^{\prime \prime}$ resolution. Activity codes: $1 . n=$ Seyfert $1 . n ; 2=$ Seyfert $2 ; 2(1)=$ hidden Seyfert 1 ; $\mathrm{L}=\mathrm{LINER} ; \mathrm{SB}=$ starburst $; \mathrm{X}=$ narrow-line $\mathrm{X}$-ray galaxy (see notes in the Appendix). Units of right ascension are hours, minutes, and seconds, and units of declination are degrees, arcminutes, and arcseconds.

${ }^{a}$ This research has made use of the NASA/IPAC Extragalactic Database (NED), which is operated by the Jet Propulsion Laboratory, California Institute of Technology, under contract with the National Aeronautics and Space Administration.

REFERENCES.-(1) de Vaucouleurs et al. 1991; (2) Brinks et al. 1997; (3) Nelson \& Whittle 1995; (4) Meaburn, Whitehead, \& Pedlar 1989; (5) Irwin \& Seaquist 1991; (6) Kenney, Carlstrom, \& Young 1993; (7) Pedlar et al. 1993; (8) Rigopoulou et al. 1996; (9) Huchra et al. 1990. 


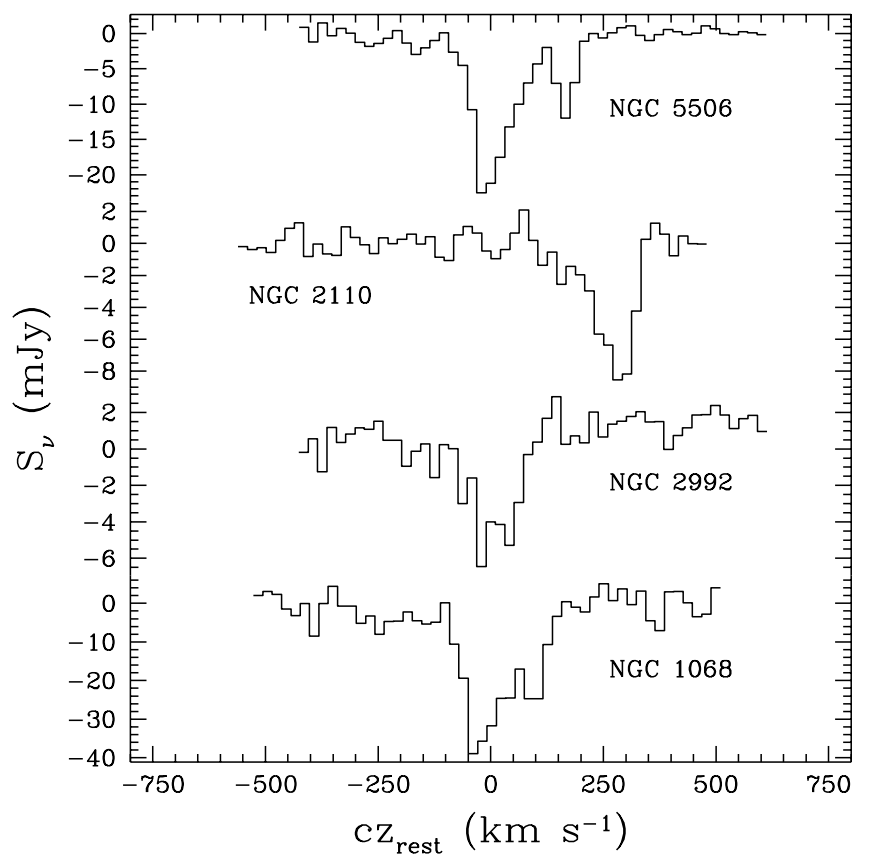

FIG. 2.-Global H I absorption spectra of Seyfert 2 and narrow-line $\mathrm{X}$-ray nuclei. The spectra are continuum-subtracted and corrected back to zero rest velocity based on the observed recessional velocities in Table 2. The nondetections are Mrk 3 and Mrk 348.

Ulvestad 1997). The Appendix details more general characteristics of these sources.

The only published survey comparable to the present one is the Dickey (1986) nonimaging VLA survey of radio bright spiral and irregular galaxies. Accepting the small number statistics, our detection rate $(69 \%)$ is better than Dickey's

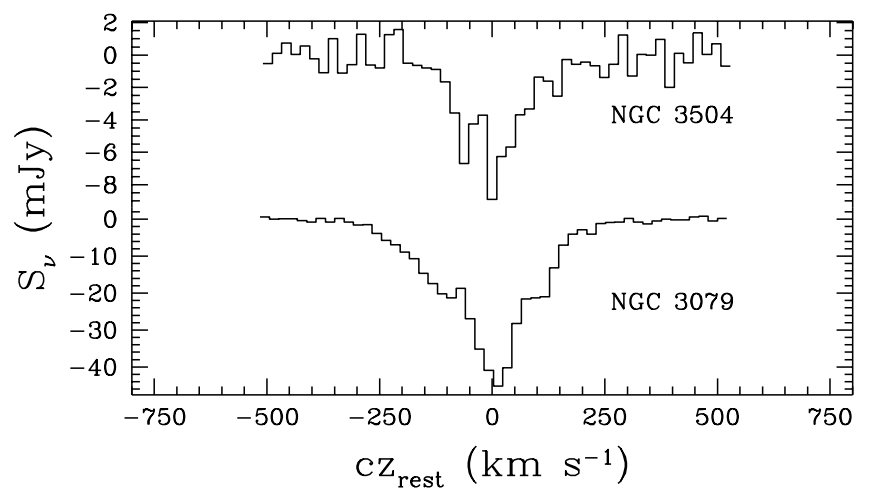

FIG. 3.-Global H I absorption spectra of starburst and LINER nuclei. The spectra are continuum-subtracted and corrected back to zero rest velocity based on the observed recessional velocities in Table 2 . The only nondetection is NGC 2639.

(53\%). The most likely explanation is a $\sim 4$ times improvement in sensitivity rather than sample selection. For example, our survey contains a greater fraction of AGNs relative to star-forming nuclei, but isolating the subsample of AGNs within Dickey's survey does not increase the detection rate. Explicitly, nineteen sources were surveyed in Dickey (1986), including 11 Seyfert or LINER nuclei and one BL Lac; rapid star formation may dominate the radio continuum emission in the remaining sources. Dickey detected 10 of $19(53 \%)$ galaxies, or less than $78 \%$ accounting for five additional, but marginal, detections. The statistics are comparable for the subsample of Seyfert and LINER nuclei: five (+ 4 marginal) of 12 were detected, for a detection rate of $42 \%(<75 \%)$. Furthermore, the distribution of continuum source brightnesses are similar between

TABLE 3

H i ABSORPTION Properties

\begin{tabular}{|c|c|c|c|c|c|c|}
\hline $\begin{array}{l}\text { Source } \\
\text { (1) }\end{array}$ & $\begin{array}{c}\text { Peak } \tau \\
\text { (2) }\end{array}$ & $\begin{array}{c}\int S_{v} d v \\
(3)\end{array}$ & $\underset{\left(\mathrm{km} \mathrm{s}^{-1}\right)}{\int \tau_{v} d v}$ & $\begin{array}{c}N_{\mathrm{HI}_{\mathrm{I}}} \\
\left(10^{21} \mathrm{~cm}^{-2}\right) \\
(5)\end{array}$ & $\begin{array}{l}\text { Separation } \\
\text { (arcsec) } \\
(6)\end{array}$ & $\begin{array}{l}\text { Projected Separation } \\
\text { (pc) } \\
(7)\end{array}$ \\
\hline Mrk $3 \ldots \ldots \ldots \ldots$ & $<0.005$ & $<0.09$ & $<0.10$ & $<0.02$ & & \\
\hline Mrk $6^{\mathrm{b}} \ldots \ldots \ldots \ldots$ & $0.45 \pm 0.01$ & $1.1 \pm 0.06$ & $14.0 \pm 1.6$ & $2.6 \pm 0.3$ & $1.0 \pm 0.10$ & $370 \pm 36$ \\
\hline Mrk $231^{\mathrm{c}} \ldots \ldots \ldots$ & $0.073 \pm 0.004$ & $3.6 \pm 0.17$ & $33.9 \pm 3.8$ & $6.3 \pm 0.7$ & $0.10 \rightarrow 0.44$ & $82 \rightarrow 360$ \\
\hline Mrk $348 \ldots \ldots \ldots$ & $<0.004$ & $<0.08$ & $<0.27$ & $<0.05$ & $\ldots$ & $\ldots$ \\
\hline Mrk $668 \ldots \ldots \ldots$ & $<0.006$ & $<0.1$ & $<0.16$ & $<0.03$ & $\ldots$ & $\ldots$ \\
\hline NGC $1068 \ldots \ldots$ & $0.078 \pm 0.004$ & $1.5 \rightarrow 2.9$ & $2.9 \rightarrow 15$ & $0.53 \rightarrow 2.73$ & $1.6 \rightarrow 8.4$ & $120 \rightarrow 625$ \\
\hline NGC $2110 \ldots \ldots$ & $0.120 \pm 0.012$ & $1.0 \pm 0.16$ & $17.3 \pm 2.1$ & $2.5 \pm 0.4$ & $1.5 \pm 0.16$ & $230 \pm 24$ \\
\hline NGC $2639 \ldots .$. & $<0.003$ & $<0.05$ & $<0.56$ & $<0.10$ & $\ldots$ & $\ldots$ \\
\hline NGC $2992 \ldots \ldots$ & $0.212 \pm 0.020$ & $1.1 \pm 0.12$ & $31.2 \pm 3.4$ & $5.7 \pm 0.6$ & $1.0 \pm 0.13$ & $152 \pm 19$ \\
\hline NGC $3504 \ldots . .$. & $0.188 \pm 0.026$ & $1.3 \pm 0.16$ & $30.2 \pm 3.0$ & $5.5 \pm 0.7$ & $<0.1$ & $<\overline{11}$ \\
\hline NGC $4151^{\mathrm{a}} \ldots .$. & $0.241 \pm 0.024$ & $0.9 \pm 0.05$ & $21.0 \pm 3.0$ & $3.8 \pm 0.6$ & $<0.1$ & $<6$ \\
\hline NGC 5506 ..... & $0.109 \pm 0.003$ & $3.0 \pm 0.10$ & $15.1 \pm 0.5$ & $2.6 \pm 0.1$ & $<0.1$ & $<11$ \\
\hline
\end{tabular}

NoTES.-Col. (1): Source ID; col. (2): peak $21 \mathrm{~cm}$ opacity; col. (3): absorption-line strength; col. (4): the velocity-integrated opacity of the strongest absorption line; col. (5): the corresponding $\mathrm{H}$ I column density, assuming $T_{S}=100 \mathrm{~K}$; col. (6): the angular separation between the AGN and the strongest $\mathrm{H}$ I absorption; col. (7): the projected separation in parsecs. Note that the measurements of $\tau$ are necessarily lower limits because of confusion and unknown covering fraction. Ranges $(\rightarrow)$ are given for the cases in which the $\mathrm{H} \mathrm{I}$ absorption is spatially resolved. For sources with unresolved $\mathrm{H}$ I absorption or continuum, the equivalent width and column density are lower limits. Expressed limits are $3 \sigma$, assuming a line width matching the $\sim 20 \mathrm{~km} \mathrm{~s}^{-1} \mathrm{channel}$ width. For the NGC 4151 upper limits on $\mathrm{H}$ I separation, we assumed that the AGN is located at C4W, the central VLBI component, after Mundell et al. 1995.

a Values include MERLIN measurements from Mundell et al. 1995.

b Values include MERLIN measurements from Gallimore et al. 1998.

${ }^{c}$ Values include VLBA measurements from Carilli et al. 1997. 
TABLE 4

H i Absorption-Line Profiles

\begin{tabular}{|c|c|c|c|c|c|c|}
\hline $\begin{array}{l}\text { Source } \\
\text { (1) }\end{array}$ & $\begin{array}{c}v_{\max } \\
\left(\mathrm{km} \mathrm{s}^{-1}\right) \\
(2)\end{array}$ & $\begin{array}{c}\text { FWHM } \\
\left(\mathrm{km} \mathrm{s}^{-1}\right) \\
(3)\end{array}$ & $\begin{array}{c}v_{50} \\
\left(\mathrm{~km} \mathrm{~s}^{-1}\right) \\
(4)\end{array}$ & $\begin{array}{c}v_{50}-v_{\max } \\
\left(\mathrm{km} \mathrm{s}^{-1}\right) \\
(5)\end{array}$ & $\begin{array}{c}v_{\max }-v_{\text {sys }} \\
\left(\mathrm{km} \mathrm{s}^{-1}\right) \\
(6)\end{array}$ & $\begin{array}{c}v_{50}-v_{\text {sys }} \\
\left(\mathrm{km} \mathrm{s}^{-1}\right) \\
(7)\end{array}$ \\
\hline Mrk $6 \ldots$ & $5572.2 \pm 0.6$ & $57.7 \pm 4.2$ & $5575.1 \pm 4.2$ & $-2.9 \pm 2.6$ & $-67.8 \pm 10.0$ & $-64.9 \pm 4.9$ \\
\hline Mrk 231 & $12664.2 \pm 5.9$ & $211.8 \pm 6.8$ & $12647.5 \pm 6.8$ & $16.7 \pm 6.5$ & $14.2 \pm 20.9$ & $-2.5 \pm 9.4$ \\
\hline NGC $1068 \ldots \ldots$ & $1117.8 \pm 1.5$ & $161.1 \pm 5.0$ & $1170.2 \pm 5.0$ & $52.4 \pm 4.7$ & $-30.2 \pm 5.2$ & $22.2 \pm 6.8$ \\
\hline NGC $2110 \ldots . .$. & $2626.3 \pm 4.3$ & $88.5 \pm 7.6$ & $2616.3 \pm 7.6$ & $10.0 \pm 5.6$ & $291.3 \pm 20.5$ & $281.3 \pm 9.4$ \\
\hline NGC $2992 \ldots \ldots$. & $2296.5 \pm 0.9$ & $95.0 \pm 4.8$ & $2326.6 \pm 4.8$ & $-30.1 \pm 4.5$ & $-17.5 \pm 6.1$ & $12.6 \pm 6.5$ \\
\hline NGC $3079 . . . .$. & $1136.2 \pm 0.4$ & $132.5 \pm 1.4$ & $1130.0 \pm 1.4$ & $6.2 \pm 1.2$ & $12.2 \pm 10.0$ & $6.0 \pm 1.8$ \\
\hline NGC $3504 \ldots . .$. & $1542.0 \pm 1.0$ & $135.5 \pm 7.4$ & $1525.3 \pm 7.4$ & $16.7 \pm 6.0$ & $8.0 \pm 2.2$ & $-8.7 \pm 9.5$ \\
\hline NGC $4151 \ldots . .$. & $994.7 \pm 0.9$ & $89.8 \pm 5.2$ & $991.0 \pm 5.2$ & $3.7 \pm 3.3$ & $-2.3 \pm 3.1$ & $-6.0 \pm 6.1$ \\
\hline NGC $5506 . . . .$. & $1802.3 \pm 0.8$ & $95.0 \pm 3.5$ & $1821.7 \pm 3.5$ & $-19.4 \pm 3.5$ & $-12.7 \pm 9.0$ & $6.7 \pm 5.0$ \\
\hline
\end{tabular}

Notes.-Col. (1): Source ID; col. (2): velocity at absorption-line minimum (i.e., peak line strength); col. (3): full-width at half-maximum; col. (4): the velocity mean across the FWHM; col. (5): as a line asymmetry parameter, the differences between $v_{\max }$ and $v_{50}$, with positive values indicating red asymmetry in the line wings; cols. (6) and (7): the difference between the systemic velocity and the line profile velocities, with positive values indicating redshift relative to systemic.

the present survey and Dickey (1986). The improved flux sensitivity of the present survey constitutes an improvement in optical depth sensitivity. An example is the starburst nucleus NGC 3504, which was detected marginally in Dickey (1986) but is clearly detected in our survey.

\section{PROPERTIES OF THE H I ABSORPTION-LINE GAS}

\subsection{Spin Temperatures}

The column density $N_{\mathrm{H} \text { I }}$ and spin (excitation) temperature $T_{S}$ are related to the observed $\mathrm{H}$ I absorption spectrum according to

$$
N_{\mathrm{HI}}=0.182 \times 10^{21}\left(T_{S} / 100 \mathrm{~K}\right) \int \tau_{v} d v \mathrm{~cm}^{-2},
$$

where $v$ is in units $\mathrm{km} \mathrm{s}^{-1}$ and $\tau_{v}$ is the opacity measured against the background continuum. In order to derive $\mathrm{H}$ I

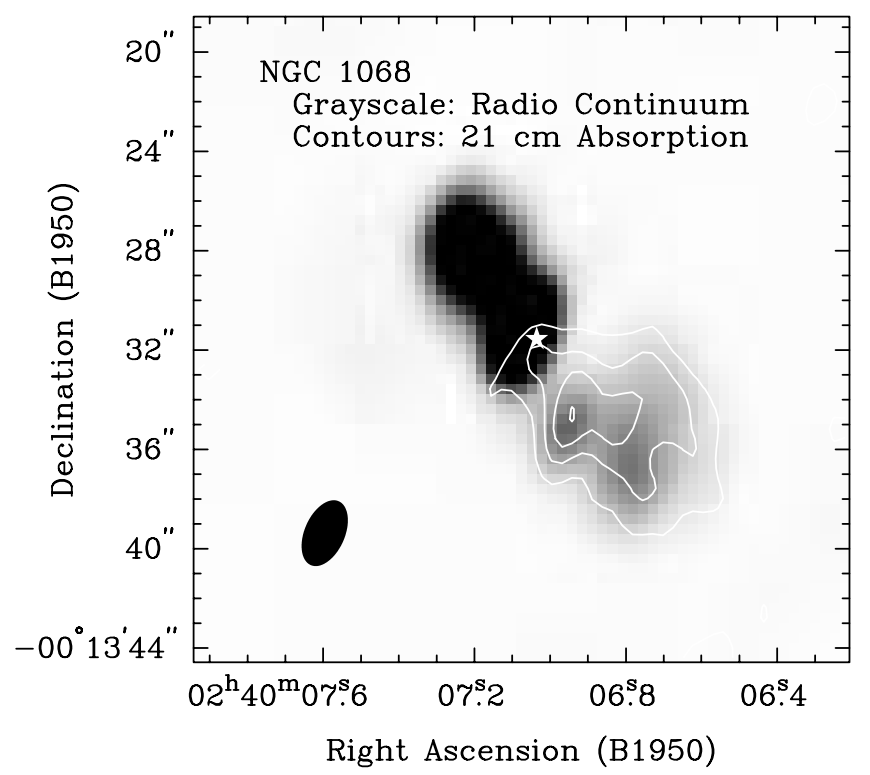

FIG. 4.-Overlay of integrated $\mathrm{H}$ I absorption (contours) on radio continuum (inverse gray scale) of NGC 1068. The contour levels are 0.21 , $-0.21,-0.41,-0.60, \&-0.80 \mathrm{Jy} \mathrm{km} \mathrm{s}^{-1}$. The maximum gray-scale level is clipped at $0.3 \mathrm{Jy}$. The star marks the location of the nucleus. The beam is indicated by the filled ellipse in the lower left-hand corner. column densities, we need some handle on the excitation state of the absorbing gas.

There are three factors controlling the spin temperature of neutral hydrogen (Field 1959a, 1959b, 1959c): collisions, pumping by Ly $\alpha$ photons, and the absorption of $21 \mathrm{~cm}$ continuum radiation. The spin temperature approaches the kinetic temperature $T_{K}$ in collisionally dominated (high density) regions. H I absorption columns through dense clouds in galaxy disks typically match the $\mathrm{H}$ I emission columns for a spin temperature of $T_{S} \sim 50-300 \mathrm{~K}$, close to the expected $T_{K}$ (e.g., Dickey, Terzian, \& Salpeter 1978; Payne, Salpeter, \& Terzian 1983; Liszt et al. 1983; Braun 1997). However, radiative effects are expected to be important in the neighborhood of an AGN, (Bahcall \& Ekers 1969 ), and $T_{K}$ is a lower limit for $T_{S}$.

Figure 12 demonstrates the effects of $21 \mathrm{~cm}$ absorption and $\mathrm{Ly} \alpha$ pumping of the ground state of $\mathrm{H} \mathrm{I}$ in the $100 \mathrm{pc}$ scale environment of a Seyfert nucleus (compare with Fig. 5 in O'Dea, Baum, \& Gallimore 1994). For illustration, we modeled the $21 \mathrm{~cm}$ radiative effects using the nuclear properties of NGC 4151. The background radio sources have a brightness temperature $T_{R} \sim 10^{6} \mathrm{~K}$ at $21 \mathrm{~cm}$ (e.g., Harrison et al. 1986; Ulvestad et al. 1998), and the specific luminosity of the continuum near Ly $\alpha$ is $F_{\mathrm{Ly} \alpha} \approx 1.8 \times 10^{27}$ ergs $\mathrm{s}^{-1} \mathrm{~Hz}^{-1}$ (Kriss et al. 1992). In computing the density of $\mathrm{Ly} \alpha$ photons we further assumed a distance of $50 \mathrm{pc}$ between the continuum source and the absorbing $\mathrm{H}$ I cloud. A distance of $50 \mathrm{pc}$ usually falls within the range of the observed, deprojected distances between the radio nuclei and $\mathrm{H} \mathrm{I}$ absorbing regions (see $\S 5$ ). We find that, in the 100 pc vicinity of an AGN, Ly $\alpha$ pumping or $21 \mathrm{~cm}$ absorption controls $T_{S}$ for densities $N_{\mathrm{HI}_{\mathrm{I}}} \lesssim 1000 \mathrm{~cm}^{-3}$, but $T_{S}$ settles asymptotically to $T_{K}$ for $n_{\mathrm{HI}} \gtrsim 1000 \mathrm{~cm}^{-3}$ as collisions dominate the excitation of the ground state.

Note that Figure 12 is representative for typical Seyfert jets. A few Seyfert galaxies, however, have bright selfabsorbed cores; Mrk 348 is an example. The intense radiation field near a self-absorbed radio core strongly affects $T_{\text {spin }}$ (e.g., Bahcall \& Ekers 1969), and this effect may suppress $\mathrm{H}$ I absorption from an obscuring torus. We consider this possibility in more detail in $\S 6.3$.

For most of the Seyfert galaxies in this survey, the $\mathrm{H} \mathrm{I}-\mathrm{absorbed}$ regions cover radii comparable, in projection, to the size of the NLR, and H I absorption might plausibly 


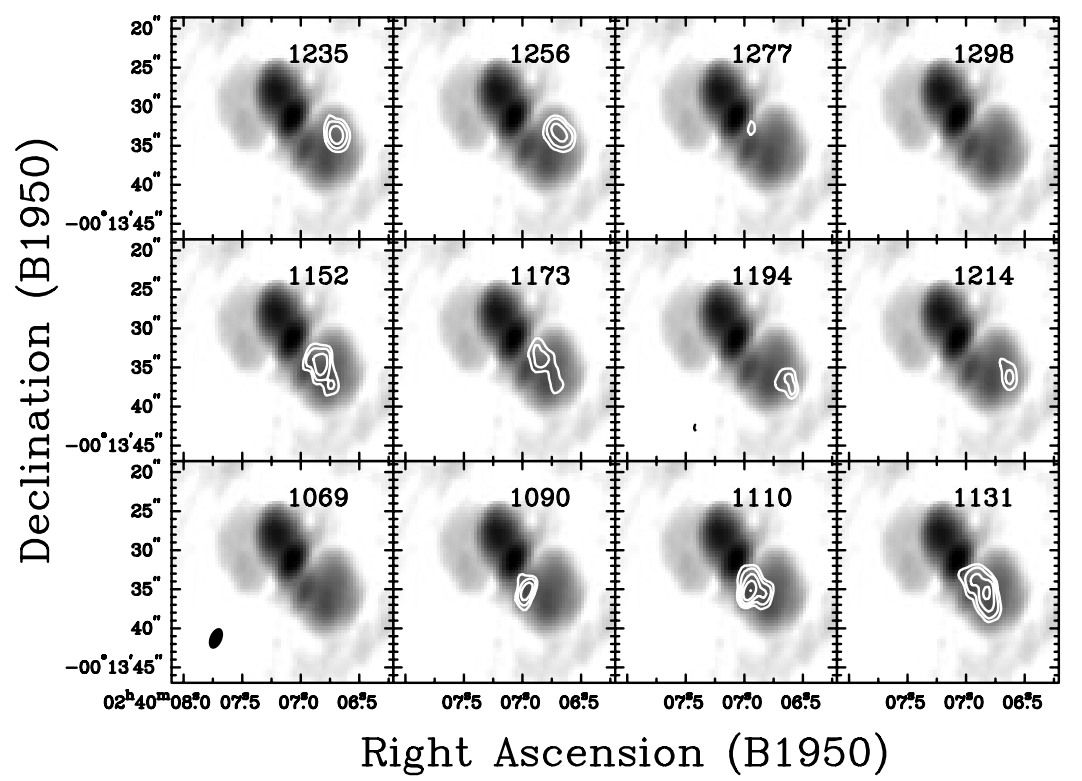

FIG. 5.-Channel-map overlays of $\mathrm{H}$ I absorption (contours) on radio continuum (inverse gray scale) of NGC 1068. The contour levels are -13.9, -9.2, $-6.1,-4.1$, and $-2.7(3 \sigma) \mathrm{mJy}_{\text {beam }}{ }^{-1}$. The gray-scale stretch is logarithmic, and the maximum gray-scale level displayed is clipped at $1.0 \mathrm{Jy}$. The channel velocities, in $\mathrm{km} \mathrm{s}^{-1}$, are provided in the upper right-hand corner of each panel.

arise from neutral filaments embedded within or shadowed by NLR plasma. Since $\tau_{v} \propto\left(T_{S} \Delta v\right)^{-1}, \mathrm{H}_{\text {I }}$ absorption studies are particularly sensitive to gas that is cold both thermally and kinematically. We would therefore expect most of the $\mathrm{H}$ I absorption to arise from the coldest regions of clouds where hydrogen is primarily atomic. In AGNirradiated clouds, the kinetic temperature of the cold atomic region is $T_{K} \approx 100 \mathrm{~K}$ (Maloney, Hollenbach, \& Tielens 1996). If we assume pressure equilibrium and typical NLR conditions $T_{e} \sim 15,000 \mathrm{~K}$ and $n_{e} \sim 2000 \mathrm{~cm}^{-3}$ (Koski 1978), the density of the cold atomic region is $n_{\mathrm{H}_{\mathrm{I}}} \sim 10^{5}$

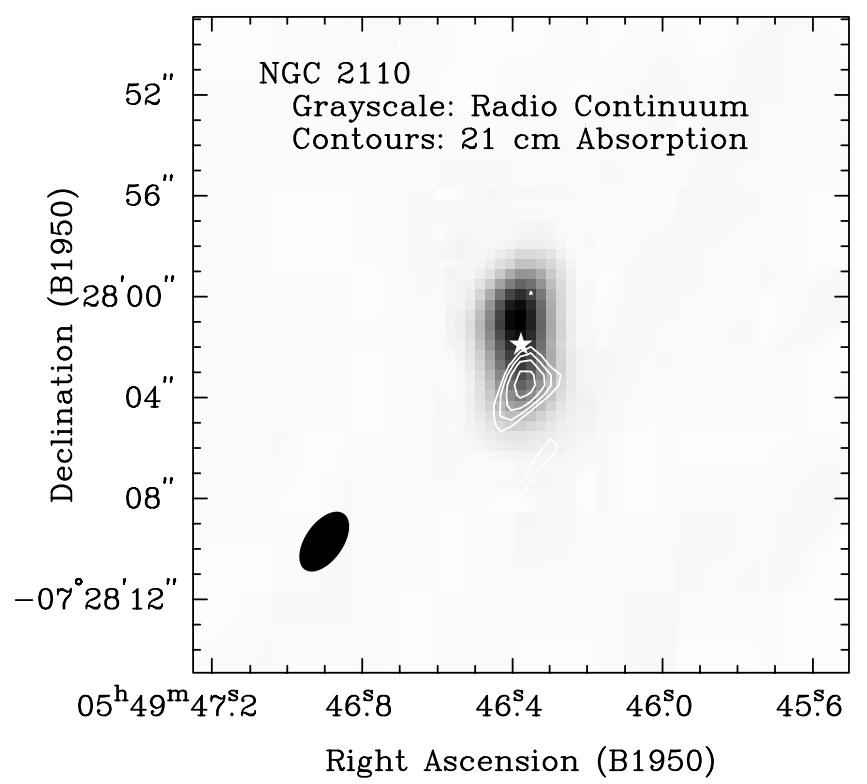

FIG. 6.-Overlay of integrated $\mathrm{H}$ I absorption (contours) on radio continuum (inverse gray scale) of NGC 2110. The contour levels are 0.45 , $-0.45,-0.57,-0.71$, and $-0.90 \mathrm{Jy} \mathrm{km} \mathrm{s}^{-1}$. The star marks the location of the radio nucleus. The beam is indicated by the filled ellipse in the lower left-hand corner. $\mathrm{cm}^{-3}$. At this limit collisions dominate, and $T_{S} \rightarrow T_{K}$ (Fig. 12).

We conclude that $\mathrm{H}$ I absorption from the NLR region of Seyfert galaxies probably arises from the cold dense regions where $T_{S} \approx T_{K} \sim 100 \mathrm{~K}$. This result is remarkable in that $T_{S}$ in cold Galactic clouds is also $\sim 100 \mathrm{~K}$, and so the conventional assumption $T_{S}=100 \mathrm{~K}$ holds up under a broad range of conditions. Of course, there may be situations in which $\mathrm{H}$ I absorption traces a warmer atomic medium in the AGN environment; for example, the sight line to the radio source may not cross the cold neutral region. In the

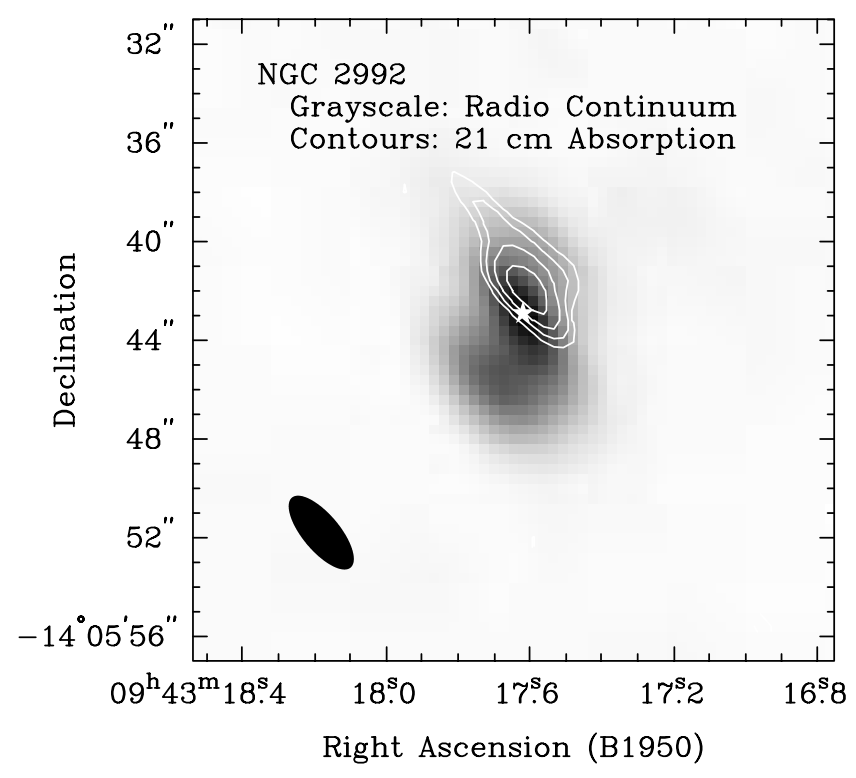

FIG. 7.- Overlay of integrated $\mathrm{H}$ I absorption (contours) on radio continuum (inverse gray scale) of NGC 2992. The contour levels are 0.36, $-0.36,-0.49,-0.66$, and $-0.90 \mathrm{Jy} \mathrm{km} \mathrm{s}^{-1}$. The star marks the location of the radio nucleus. The beam is indicated by the filled ellipse in the lower left-hand corner. 


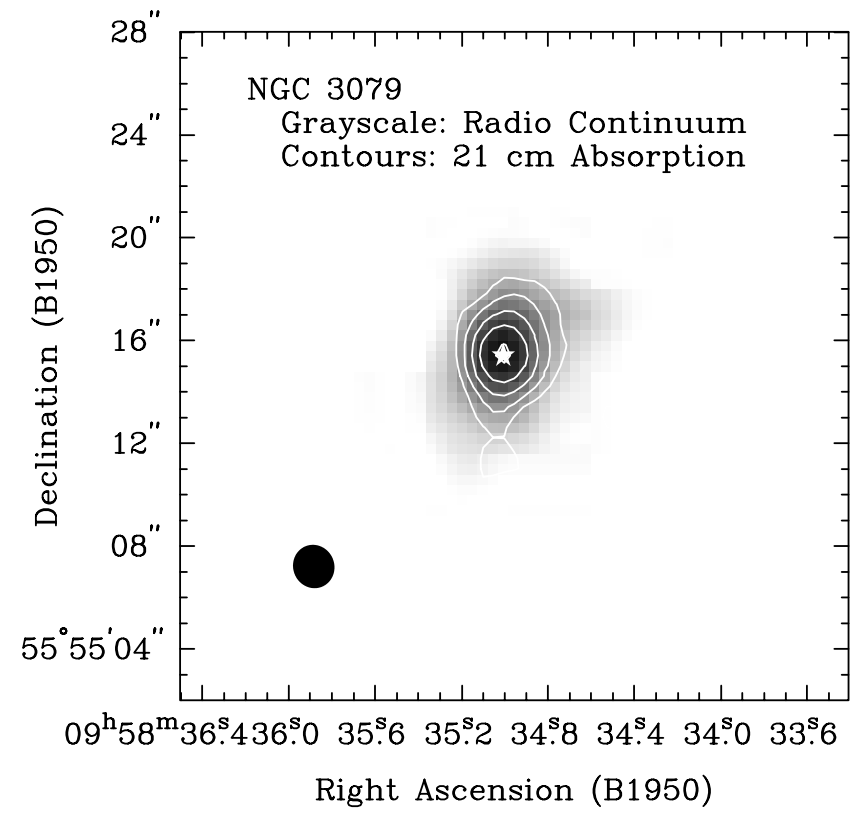

FIG. 8.-Overlay of integrated $\mathrm{H} \mathrm{I}$ absorption (contours) on radio continuum (inverse gray scale) of NGC 3079. The contour levels are 0.57 , $-0.57,-1.1,-2.2,-4.3$, and $-8.4 \mathrm{Jy} \mathrm{km} \mathrm{s}^{-1}$. The continuum is displayed with a logarithmic stretch. The star marks the peak of the radio continuum, which is presumably the nucleus. The beam is indicated by the filled ellipse in the lower left-hand corner.

warm atomic region $T_{K}$ (and $T_{S}$ ) can rise to several $1000 \mathrm{~K}$ before hydrogen is significantly ionized (Maloney et al. 1996). The assumption of $T_{S}=100 \mathrm{~K}$ therefore provides a lower limit to the true $\mathrm{H}$ I column.

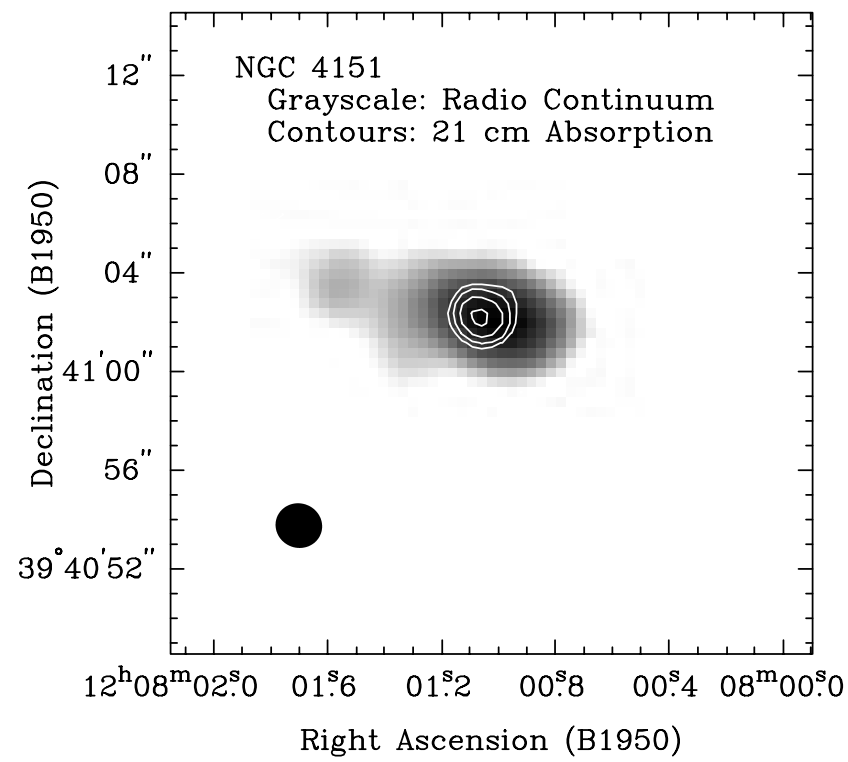

FIG. 10.- Overlay of integrated $\mathrm{H}$ I absorption (contours) on radio continuum (inverse gray scale) of NGC 4151 . The contour levels are $0.3,-0.3$, $-0.45,-0.70$, and $-1.1 \mathrm{Jy} \mathrm{km} \mathrm{s}^{-1}$. The beam is indicated by the filled ellipse in the lower left corner.

\subsection{Column Densities}

We derived the column densities of the absorbing $\mathrm{H}$ I according to equation (1) and assuming a spin temperature $T_{S}=100 \mathrm{~K}$. The measured columns are listed in Table 3 . The absorption opacity $\tau$ was measured against channelaveraged continuum images from the same data set. Confusion between continuum sources can dilute the measured

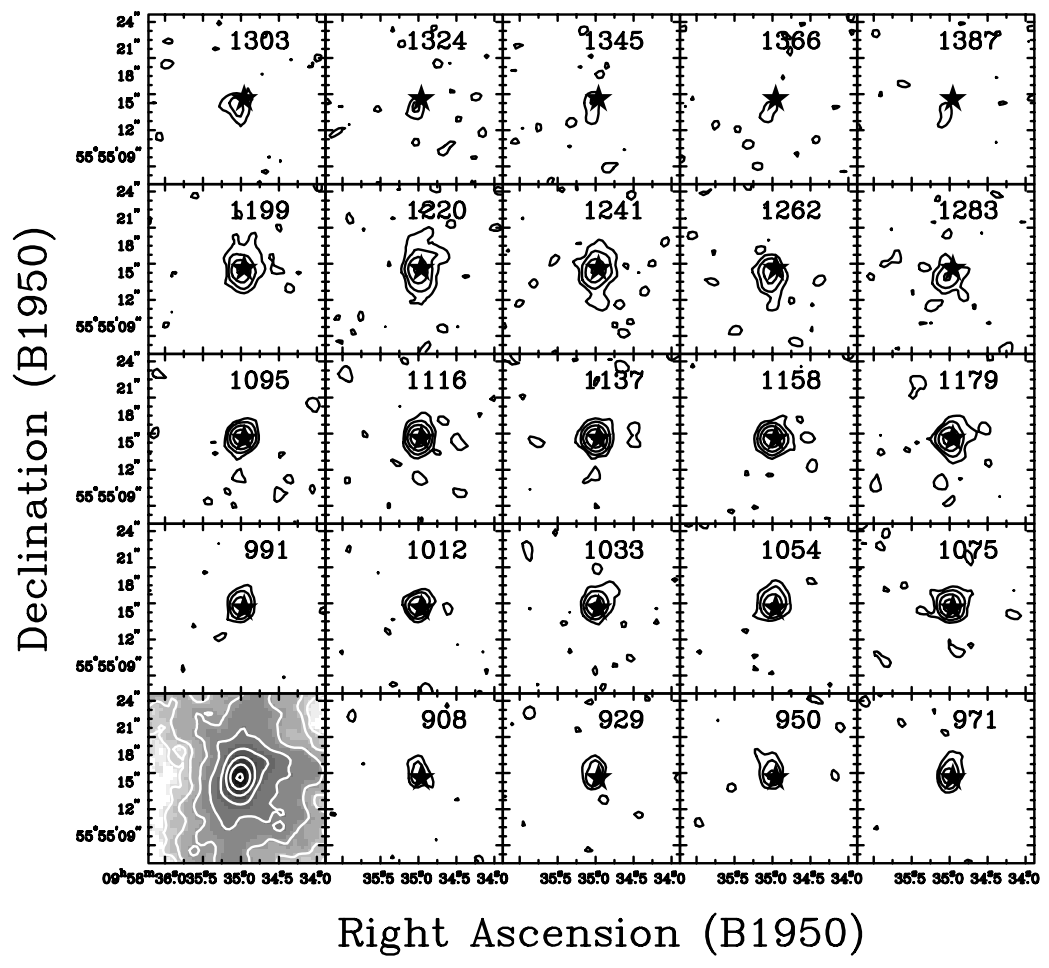

Fig. 9. - Channel maps of the H I absorption (contours) toward NGC 3079. The contour levels are $-40.5,-19.1,-9.0,-4.2,-2$, and $-2 \mathrm{mJy}$ beam ${ }^{-1}$. The continuum is displayed as contours on gray scale in the lower left-hand panel. The star symbol marks the location of the radio continuum peak on the channel maps. The continuum contour levels are $-0.30,0.30,0.66,1.5,3.2,7.0,15.4,34.0$, and $74.7 \mathrm{mJy} \mathrm{beam}^{-1}$. The channel velocities, in $\mathrm{km} \mathrm{s}^{-1}$, are provided in the upper right-hand corner of each panel. A $\sim 0$.' 3 scale velocity gradient, with velocities increasing from northwest through southeast, is marginally resolved by our data (compare with Pedlar et al. 1996). 


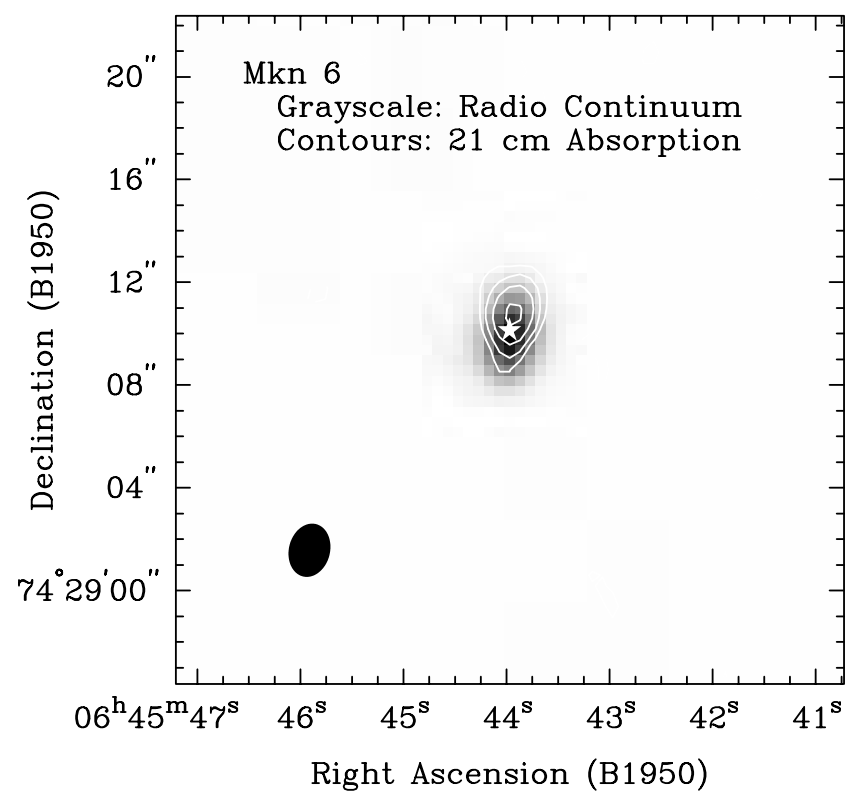

FIG. 11. - Overlay of integrated $\mathrm{H}$ I absorption (contours) on radio continuum (inverse gray scale) of Mrk 6 . The contour levels are $0.19,-0.19$, $-0.46,-1.1$, and $-2.7 \mathrm{Jy} \mathrm{km} \mathrm{s}^{-1}$. The star marks the location of the optical nucleus (Clements 1981). The beam is indicated by the filled ellipse in the lower left-hand corner.

opacities or, equivalently, the $\mathrm{H}$ I absorbing gas might not completely cover an unresolved background source. The reported values of $N_{\mathrm{H}_{\mathrm{I}}}$ are therefore lower limits to the true absorbing column. The columns for Mrk 6, NGC 4151, and

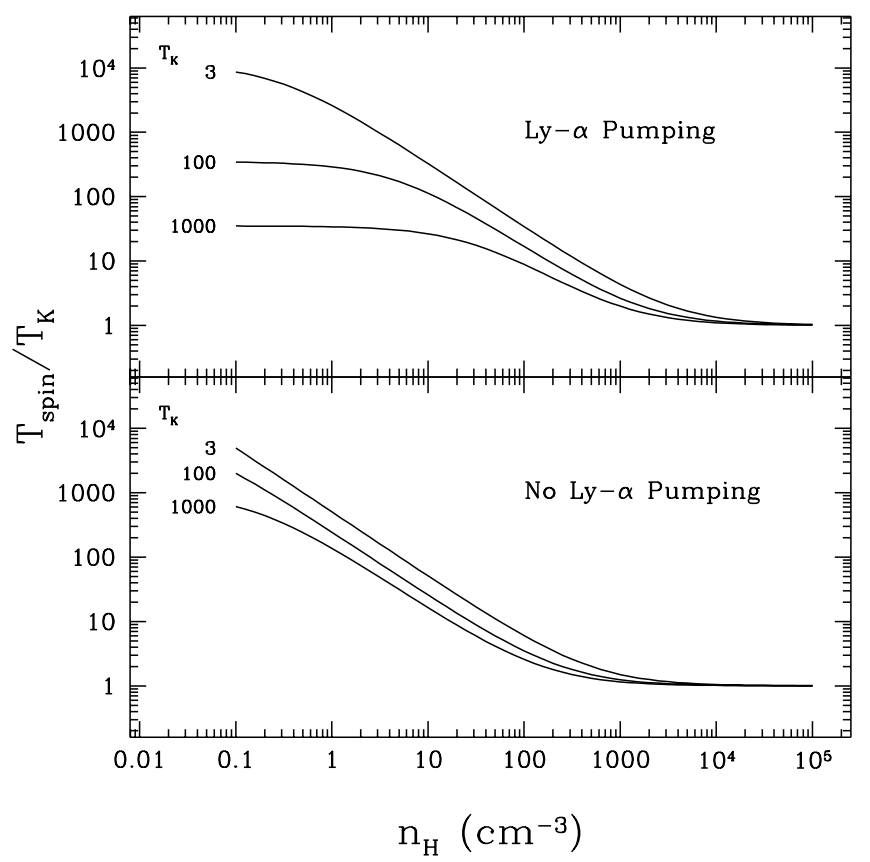

FIG. 12.-Plots of $T_{S}(21 \mathrm{~cm}) / T_{K}$ for varying kinetic temperature $T_{K}$ as a function of neutral hydrogen density $n_{\mathrm{H}}$. The models plotted in the bottom panel include only the effects of $21 \mathrm{~cm}$ absorption and collisional excitation, but the plots in the top panel include the effects of Ly $\alpha$ pumping of the ground state of $\mathrm{H}$ I. The three curves on each panel correspond to $T_{K}=3$, 10 , and $100 \mathrm{~K}$. We modeled the radio and UV continuum properties after NGC 4151, assuming a distance of 50 pc between the AGN and absorbing cloud (further details are provided in the text). Irrespective of the presence of a UV pump, the $21 \mathrm{~cm}$ transition thermalizes for $n_{\mathrm{H}} \gtrsim 1000 \mathrm{~cm}^{-3}$. Note that $21 \mathrm{~cm}$ continuum excitation may affect $T_{\text {spin }}$ in Mrk 348 and other sources with bright, flat-spectrum cores (see $\S 6.3$ ).
Mrk 231 are derived from published MERLIN and VLBA observations (Mundell et al. 1995; Carilli et al. 1997; Gallimore et al. 1998).

One concern of this study is whether the $\mathrm{H} \mathrm{I}$ absorbing gas is peculiar to the small-scale AGN environment or arises from the disk of the host galaxy on larger scales. Addressing this question, a simple prediction would be that the $\mathrm{H}$ I absorption should show no trend with inclination, since AGN disks appear to be oriented randomly with respect to outer galaxy disks (e.g., Schmitt et al. 1997; Nagar et al. 1999). Figure 13 compares the distribution of $\mathrm{H} \mathrm{I}$ columns with the inclination of the host galaxy, estimated from optical axial ratios tabulated by de Vaucouleurs et al. (1991; compare with Figure 5 in Dickey 1986). Figure 13 also includes $\mathrm{H}$ I absorption measurements from this work and Dickey (1986). Only those sources with well-defined disk morphology and optical axial ratio are plotted. For reference, we also plot the range of Galactic $\mathrm{H}$ I absorbing columns observed toward extragalactic radio sources (Payne, Salpeter, \& Terzian 1982) and the radio source Sgr A in the Galactic center (Schwarz, Ekers, \& Goss 1982).

We can draw two conclusions from Figure 13. First, running counter to the prediction for AGN disks, there is evidence for a trend in $\mathrm{H}$ I column with host galaxy inclination. For instance, all four sources with $i>60^{\circ}$ are detected in $\mathrm{H}$ I absorption. We computed correlation statistics for the regression of $N_{\mathrm{H}_{\mathrm{I}}}$ on $\sec i$ using standard survival analysis techniques to account for the nondetections. The results indicate a real trend in $n_{\mathrm{H}}$ with $i$ : the probability for

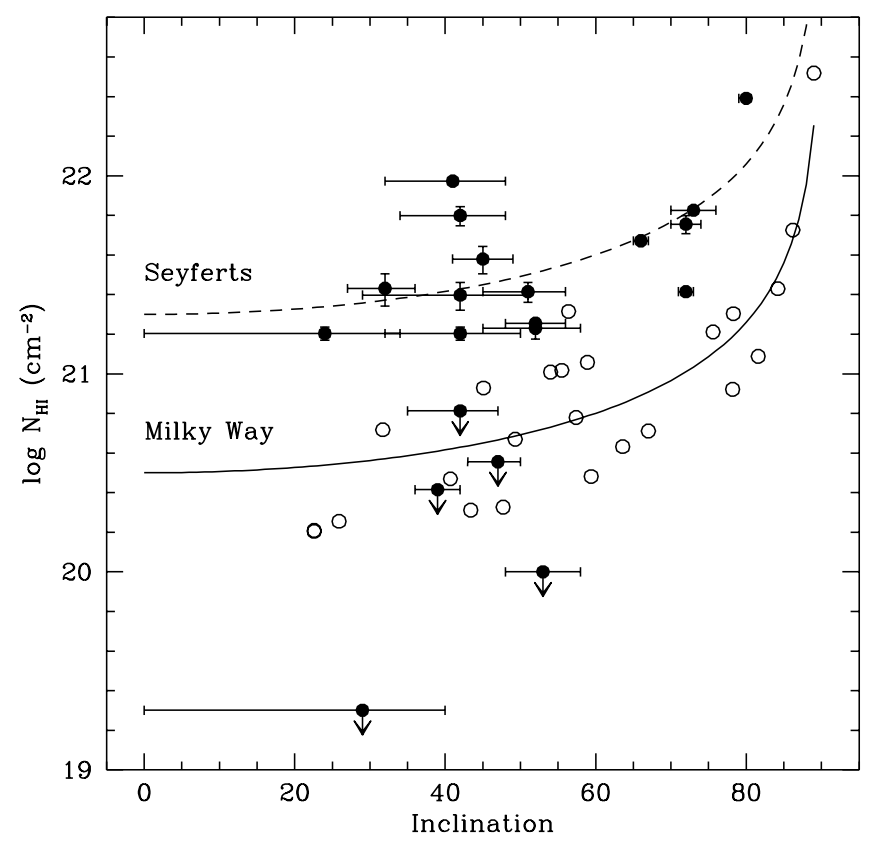

FIG. 13.-Absorbing $\mathrm{H}$ I column density plotted vs. galaxy inclination. The columns for Seyfert galaxies are plotted as filled circles; upper limits are indicated by arrows. Measurements from Dickey (1986) have been included. The Seyfert columns assume a characteristic $T_{\text {spin }}=100 \mathrm{~K}$, which may be a lower limit to the true spin temperature. The dashed line traces the secant curve appropriate for a face-on column density of $2 \times 10^{21}$ $\mathrm{cm}^{-2}$, representative of the Seyfert detections. The open circles mark Galactic absorption data from Payne et al. (1982) and Schwarz et al. (1982). The Galaxy absorption columns have been corrected for measured $T_{\text {spin }}$. The effective inclination of the Galaxy absorption measurements was taken to be the complement of the Galactic latitude. The mean face-on column for the Galactic absorption measurements is $3 \times 10^{20} \mathrm{~cm}^{-2}$ and is traced by the solid secant curve. Compare with Fig. 5 of Dickey (1986). 
no correlation is $2.7 \%$ for Kendall's generalized $\tau,<1 \%$ for Cox's proportional hazard model, and $4.6 \%$ for Spearman's generalized $\rho$ (although we caution that Spearman's $\rho$ is not reliable for sample sizes $\lesssim 30$ ). This result suggests that in many Seyfert galaxies $\mathrm{H}$ I absorption arises in gaseous disks at least aligned with, if not cospatial with, the kiloparsecscale galaxy disks. We caution that the trend is valid only for the quantity $N_{\mathrm{H}} / T_{\text {spin }}$, but a significant spread in $T_{\text {spin }}$ would reduce confidence in the trend of $N_{\mathrm{H}_{\mathrm{I}}}$ with inclination. Nevertheless, we demonstrate in $\S 5$ that the kinematics and distribution of the $\mathrm{H} \mathrm{I}$ absorption-line gas are also consistent with the observed trend.

The second conclusion is that the typical absorbing columns toward detected Seyfert galaxies exceed the Galactic columns by factors ranging from $\sim 3$ to 30 , although there is some overlap for individual Seyfert galaxies and Galactic absorbing clouds. The mean face-on column for detected Seyfert galaxies is $4 \times 10^{21} \mathrm{~cm}^{-2}$, exceeding Galaxy columns by a factor of nearly 10 . It would be interesting to compare the Seyfert $\mathrm{H}$ I columns with a larger sample of nonactive galaxies, but, lacking bright radio sources, nonactive galaxies are not readily studied in $\mathrm{H} \mathrm{I}$ absorption. Nevertheless, based on $\mathrm{H}$ I emission studies, the face-on columns through spiral galaxies are typically a few times $10^{20} \mathrm{~cm}^{-2}$ (e.g., surveys include the theses of Bosma 1978; Begeman 1987; van Driel 1987; their associated publications; and Broeils \& van Woerden 1994). Typical H I emission columns of normal spiral galaxies are comparable to the absorption columns measured for the Galaxy rotated to a face-on view.

The observed enhancement of H I column toward Seyfert nuclei is probably not an excitation effect. Demonstrated above in $\S 4.1$, the measured columns are lower limits because $21 \mathrm{~cm}$ absorption and Ly $\alpha$ pumping in the vicinity of an AGN can raise $T_{S}$ to $1000 \mathrm{~K}$ or more. In contrast all of the Galactic values have been corrected for directly measured spin temperatures (for the Galactic center column we assumed $T_{S} \approx T_{K} \sim 300 \mathrm{~K}$ in the $10 \mathrm{pc}$ diameter disk, after Genzel et al. 1985, and references therein). Furthermore, owing to proximity, $\mathrm{H}$ I absorbing clouds in the Galaxy are better resolved compared to H I absorption toward Seyfert nuclei. Therefore, the Galaxy columns suffer less continuum dilution owing to confusion or covering fraction arguments. We conservatively conclude that the $\mathrm{H}$ I absorption columns toward Seyfert nuclei exceed the $\mathrm{H}$ I columns in the Galaxy, and perhaps other nonactive spiral galaxies, by a factor of at least 6 (as shown in Fig. 13).

We show in $\S 5$ that $\mathrm{H}$ I absorption in Seyfert galaxies usually traces $\mathrm{H}$ I disks in the inner kiloparsec. In contrast, $\mathrm{H}$ I columns are often reduced below detectability in the inner kiloparsec of nearby, nonactive spirals (e.g., Roberts 1975; Giovanelli \& Haynes 1988). Limited by instrumental sensitivity, the central columns are typically $\lesssim C_{f} \times 10^{20}$ $\mathrm{cm}^{-2}$, where $C_{f}$ is the beam covering fraction of the $\mathrm{H}$ i gas. The high detection rate for Seyfert nuclei argues for $C_{f}$ approaching unity. Based on the interpretation of $\S 5$, it appears that the $\mathrm{H}$ I surface density in the inner kiloparsec of Seyfert and starburst galaxies exceeds by a factor $\gtrsim 20$ the $\mathrm{H}$ I columns in the centers of nonactive galaxies. We discuss this result further in $\S 7$.

\subsection{Comparison with $X$-Ray Columns}

$\mathrm{X}$-rays provide another measure of the column of material obscuring the central engine in active galaxies.
Photoelectric absorption by foreground gas that is not fully ionized attenuates the soft X-ray continuum spectrum of the central engine. The simplest models for AGN X-ray spectra usually involve a power-law continuum modified by photoelectric absorption through the Galaxy and photoelectric absorption intrinsic to the source. The absorption is commonly parameterized by a total hydrogen column adopting standard cross sections and abundances (e.g., Morrison \& McCammon 1983).

Table 5 lists the X-ray absorption columns available in the literature for sources in the combined survey. There are often several models for a given source, and predictably the absorption column becomes poorly constrained as models become more complex. In each case, we selected the simplest model that provides a reasonable fit and the best constraints on an absorption column. Selecting models in this way tended to err on the side of caution; that is, more sophisticated models often give larger measures for the $\mathrm{X}$-ray photoelectric column. The conclusions of this section are not affected by the selection of the simplest X-ray spectral models.

Figure 14 compares the $21 \mathrm{~cm}$ absorption columns with the X-ray absorption columns. Under the hypothesis that the radio and $\mathrm{X}$-ray nuclei are effectively cospatial, the prediction is a correlation between the $21 \mathrm{~cm}$ and X-ray columns. In addition, one would also expect that the X-ray columns should tend to be slightly greater than the $21 \mathrm{~cm}$ columns, since $21 \mathrm{~cm}$ absorption is not sensitive to the presence of hydrogen locked in molecules or partially ionized gas.

We find no measurable correlation between $21 \mathrm{~cm}$ and $\mathrm{X}$-ray absorption columns, and the columns match reasonably only for Mrk 231. For most (eight of 11) of the sources, the X-ray column exceeds the $21 \mathrm{~cm}$ column by at least an order of magnitude. We refer to these sources as having a "photoelectric excess" of absorption. There are three explanations for photoelectric excesses. First, it may be that most

TABLE 5

Soft X-RAY-Absorbing Columns

\begin{tabular}{|c|c|c|}
\hline Source & $\begin{array}{c}N_{\mathrm{H}} \\
\left(10^{21} \mathrm{~cm}^{-2}\right)\end{array}$ & Reference \\
\hline Mrk $3 \ldots \ldots \ldots$ & $707.9_{-47}^{+51}$ & 1 \\
\hline Mrk $6 \ldots \ldots \ldots \ldots$ & $38.0_{-0.6}^{+5.0}$ & 2 \\
\hline Mrk $231 \ldots \ldots \ldots$ & $5.3_{-3.3}^{+3.3}$ & 3 \\
\hline Mrk $348 \ldots \ldots \ldots$ & $112.2_{-21}^{+20}$ & 1 \\
\hline Mrk $501 \ldots \ldots \ldots$ & $<18$ & 4 \\
\hline NGC $1068 \ldots \ldots$ & $>1000$ & 5 \\
\hline NGC $2110 \ldots \ldots$ & $28.4_{-1.6}^{+1.9}$ & 6 \\
\hline NGC $2992 . . . . .$. & $1.3_{-0.7}^{+1.6}$ & 6 \\
\hline NGC $3079 . . . .$. & $0.6_{-0.1}^{+0.1}$ & 7 \\
\hline NGC $3227 \ldots \ldots$ & $5.0_{-0.9}^{+0.9}$ & 8 \\
\hline NGC $4151 \ldots . .$. & $22.6_{-1.0}^{+1.0}$ & 8 \\
\hline NGC $5506 \ldots \ldots$ & $21.9_{-2.4}^{+2.6}$ & 9 \\
\hline
\end{tabular}

Note.-For those cases in which more than one X-ray spectral model is available in the literature, we selected the model giving a reasonable fit to the data and the best constraint on the hydrogen column.

REFERENCES. - (1) Mulchaey et al. 1992; (2) Feldmeier et al. 1999; (3) Wang, Brinkmann, \& Bergeron 1996; (4) Mushotzky et al. 1978; (5) Matt et al. 1997, and references therein; (6) Turner et al. 1997; (7) Read et al. 1997; (8) George et al. 1998; (9) Mulchaey et al. 1993. 


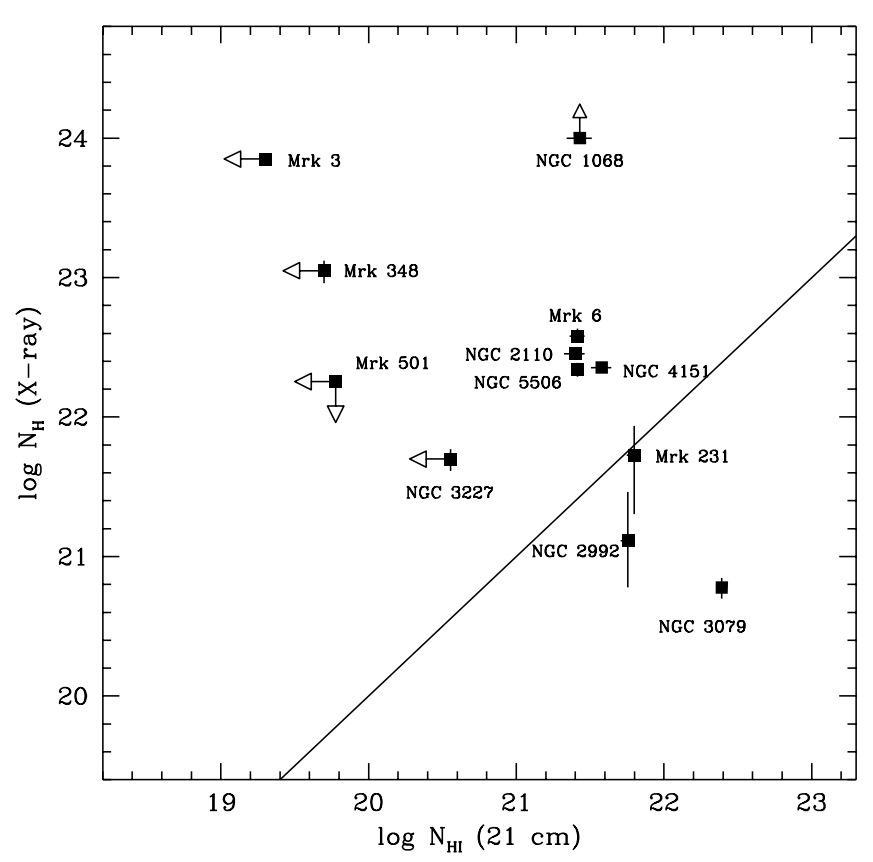

FIG. 14.-Comparison of $\mathrm{H}$ I columns measured by $21 \mathrm{~cm}$ absorption ( $x$-axis) with total hydrogen columns measured by model fits to soft X-ray spectra ( $y$-axis). We assumed $T_{\text {spin }}=100 \mathrm{~K}$ in deriving $N_{\mathrm{H}}$; the $N_{\mathrm{H}_{\mathrm{I}}}$ measurements are actually lower limits. Narrow-line objects, including Seyfert type 2, LINER, and starburst nuclei, are marked by filled squares, and broad-line objects are marked by unfilled squares. The line traces $N_{\mathrm{HI}_{\mathrm{I}}}(21$ $\mathrm{cm})=N_{\mathrm{H}}(\mathrm{X}$-ray).

of the hydrogen is in molecular or ionized form. Alternatively, it may be that the primary X-ray absorber is dust, and the obscuring material has a lower gas-to-dust ratio than assumed in the modeling. The former explanation seems most likely for the Seyfert 1 nuclei, in which it appears that the absorbing medium is ionized (e.g., George et al. 1998; Feldmeier et al. 1999). The second explanation is that the radio emission may not be cospatial with the nuclear X-ray source, and so X-ray absorption and $21 \mathrm{~cm}$ absorption may probe different regions of the foreground media. We consider this possibility further in $\S 6$. The third explanation might be spin-temperature effects. Increasing $T_{\text {spin }}$ to $\sim 1000 \mathrm{~K}$ would produce a better agreement between X-ray and $21 \mathrm{~cm}$ columns for Mrk 6, NGC 2110, NGC 4151, and NGC 5506, and the remaining photoelectric excess sources require $T_{\text {spin }}>10^{4} \mathrm{~K}$ to explain the discrepancy. This explanation fails, however, to explain the result that $\mathrm{H}$ I absorption tends to avoid the nucleus in those Seyfert galaxies where the radio jet is resolved $(\S 5$, below). It seems more likely that $21 \mathrm{~cm}$ absorption does not trace the same gas giving rise to the soft X-ray opacity.

The sources NGC 2992 and NGC 3079 show a "photoelectric deficit," meaning that the $21 \mathrm{~cm}$ absorption column exceeds the X-ray photoelectric column. Again, it may be that the abundances assumed for the X-ray modeling are incorrect. For example, in this case, the gas-to-dust ratio may be higher than was assumed for the X-ray spectral modeling. The host galaxies of both sources are edgeon, and both show evidence for extended soft X-ray emission (e.g., Elvis et al. 1990; Read, Ponman, \& Strickland 1997; Colbert, et al. 1998). The spatial resolution of the soft X-ray spectra is very coarse, $\sim 30^{\prime}$, much larger than the VLA beam. An alternative explanation, then, might be that extended X-ray emission dilutes the soft X-ray columns toward the nucleus. The extended emission that is resolved by current X-ray satellites is only $\sim 10 \%-20 \%$ of the total, insufficient to explain the photoelectric deficits. Dilution of the soft X-ray columns would require extended X-ray emission on subarcminute scales not yet resolved; future X-ray satellites will have sufficient resolution to test the hypothesis.

We conclude that there is no correlation between X-ray photoelectric columns and $21 \mathrm{~cm}$ columns. Although there are a number of possible explanations, the simplest explanation may be that the radio and X-ray sources are not cospatial, and so $21 \mathrm{~cm}$ absorption and soft X-ray absorption do not (usually) trace the same foreground gas. We consider the implications in more detail in the following discussion (particularly $\S 6$, below).

\section{DISK MODELS FOR THE DISTRIBUTION AND KINEMATICS OF THE H I ABSORPTION}

There are now a handful of Seyfert nuclei in which the $\mathrm{H}$ I absorption is resolved against the background radio source: Mrk 6 (this study; Gallimore et al. 1998), Mrk 231 (Carilli et al. 1997), NGC 1068 (this study, Fig. 4; Gallimore et al. 1994), NGC 2110 (this study, Fig. 6), NGC 2992 (this study, Fig. 6), NGC 4151 (this study, Fig. 10); Mundell et al. 1995), \& NGC 5929 (Cole et al. 1998). H I absorption is also resolved in the starburst nucleus of NGC 3079 (this study; Pedlar et al. 1996; Satoh, Inoue, \& Nakai 1998). The remaining detections that we have imaged, but in which the $\mathrm{H} \mathrm{I}$ absorption is not well resolved against the background continuum, are NGC 3504 and NGC 5506.

It is remarkable that the $\mathrm{H} \mathrm{I}$ absorption tends to avoid the radio nucleus. More typically, the absorption is displaced by $\gtrsim 100 \mathrm{pc}$ in projection away from the central radio source (Table 3). The exceptions are NGC 3079 and NGC 5506, which are edge-on, and NGC 4151, in which the $\mathrm{H}$ I absorbing gas lies within a few tens of parsecs of the central engine (Mundell et al. 1995). We have demonstrated that detections are more likely with increasing inclination of the host galaxy (§ 4.2; Fig. 13; see also Dickey 1986). Moreover, the inferred absorption columns are not correlated with estimates of the X-ray absorption column ( $\S 4.3)$. It appears that $\mathrm{H}$ I absorption in Seyfert and starburst nuclei traces gas in the inner disk of the host galaxy rather than gas associated with the AGN. In particular, the H I absorption does not appear to be associated with a parsec-scale obscuring torus, with NGC 4151 being an important exception.

To test this conclusion, we modeled the kinematics of the $\mathrm{H} \mathrm{I}$ absorbing gas as disks in circular rotation. We spatially resolve the kinematics only in NGC 1068 (Gallimore et al. 1994) and so had to fit disk models to global absorptionline profiles rather than spatially resolved channel maps. The results are listed in Table 6 and displayed in Figures $15-23$.

\subsection{Procedure}

The disk models were computed and fit as follows. First, we generated a background continuum model from the continuum CLEAN components. For NGC 4151 we constructed a continuum model based on VLBI observations (Ulvestad et al. 1998), which recover all of the continuum flux in the MERLIN maps around the region of the $\mathrm{H} \mathrm{I}$ absorption (Mundell et al. 1995). We next generated a model disk described by vertical column density $N_{\mathrm{H}}$; rota- 
TABLE 6

Model Disk Parameters

\begin{tabular}{|c|c|c|c|c|c|c|c|c|c|}
\hline $\begin{array}{l}\text { Source } \\
\text { (1) }\end{array}$ & $\begin{array}{c}i \\
(\mathrm{deg}) \\
(2)\end{array}$ & $\begin{array}{l}\text { P.A. } \\
\text { (deg) } \\
(3)\end{array}$ & $\begin{array}{l}\text { References for } \\
\text { Kinematic Axes } \\
\text { (4) }\end{array}$ & $\begin{array}{c}v_{\text {rot }} \\
\left(\mathrm{km} \mathrm{s}^{-1}\right) \\
(5)\end{array}$ & $\begin{array}{c}N_{\mathrm{HI}_{\mathrm{I}}}(\text { Face-on }) \\
\left(\times 10^{21} \mathrm{~cm}^{-2}\right) \\
(6)\end{array}$ & $\begin{array}{l}r_{\text {in }} \\
(\mathrm{pc}) \\
(7)\end{array}$ & $\begin{array}{l}r_{\text {out }} \\
\text { (pc) } \\
(8)\end{array}$ & $\begin{array}{c}\log M_{\mathrm{HI}} \\
\left(M_{\odot}\right) \\
(9)\end{array}$ & $\begin{array}{l}\chi^{2} \\
(10)\end{array}$ \\
\hline Mrk $6 \ldots \ldots \ldots \ldots$ & 51 & 130 & 1 & $140 \pm 4$ & $1.65 \pm 0.07$ & 293 & 426 & 6.60 & 143.8 \\
\hline NGC $1068 \ldots \ldots$. & 40 & -45 & 2 & $320 \pm 82$ & $0.82 \pm 0.056$ & 212 & 957 & 7.26 & 64.5 \\
\hline NGC $2110 \ldots \ldots$. & 53 & 161 & 3 & $412 \pm 5$ & $2.31 \pm 0.25$ & 250 & 552 & 7.15 & 57.0 \\
\hline NGC $2992 \ldots . . .$. & 70 & -154 & 4 & $147 \pm 8$ & $0.73 \pm 0.05$ & $36 \pm 34$ & 921 & 7.20 & 185.9 \\
\hline NGC $3079 \ldots \ldots$. & 84 & 166 & 5 & $177 \pm 1$ & $1.53 \pm 0.01$ & $8 \pm 1$ & $282 \pm 4$ & 6.48 & 1089.0 \\
\hline NGC $3504 \ldots \ldots .$. & 22 & -33 & 6 & $235 \pm 27$ & $2.33 \pm 0.21$ & $8 \pm 1$ & $<312$ & $<6.76$ & 50.5 \\
\hline NGC $4151^{a} \ldots \ldots$ & 21 & 22 & 7 & $-148 \pm 8$ & $9.64 \pm 0.63$ & $\ldots$ & 7 & 4.85 & 157.8 \\
\hline NGC $4151^{b} \ldots \ldots$ & 50 & 180 & 8 & $839 \pm 122$ & $10.20 \pm 0.69$ & $\ldots$ & 10 & 4.87 & 66.6 \\
\hline NGC $5506 \ldots \ldots$. & 80 & -87 & 9 & $181 \pm 5$ & $0.25 \pm 0.01$ & $7 \pm 2$ & $406 \pm 26$ & 6.00 & 963.2 \\
\hline
\end{tabular}

Notes.-Col. (1): Source ID; col. (2): inclination; col. (3): position angle to receding major axis; col. (4): references for the kinematic axes; col. (5): the deprojected rotational velocity at $100 \mathrm{pc}$ radius; col. (6): face-on column density through the disk; col. (7): the deprojected inner radius; col. (8): the deprojected outer radius; col. (9): logarithm of the $\mathrm{H}$ I mass (in $M_{\odot}$ ) assuming a uniform and symmetric disk; col. (10): $\chi^{2}$. Error bars are given for fitted parameters. Where not explicitly fit, the inner and outer radii span the deprojected range of the observed absorption. The reported $\chi^{2}$ values are for 50 spectral data points and three to five fitted parameters. We fit the MERLIN data of Gallimore et al. 1998 for Mrk 6; the $\chi^{2}$ values are for 53 spectral data points and three fitted parameters. Note that the $\mathrm{H}$ I masses of the model disks are less than $0.2 \%$ of the dynamical mass at the corresponding radii. References for the kinematic axes are, Mrk 6:

${ }^{a}$ The inclination and position angle are fixed to those of the outer galaxy disk.

${ }^{b}$ The inclination and position angle are fixed to those predicted for an AGN disk (i.e., perpendicular to the radio jet).

REFERENCES FOR KINEMATIC AXES.- (1) Meaburn et al. 1989; (2) Brinks et al. 1997 and Gallimore et al. 1994; (3) Wilson \& Baldwin 1985; (4) Colina et al. 1987; (5) Irwin \& Seaquist 1991; (6) Kenney et al. 1993; (7) Pedlar et al. 1992; (8) Mundell et al. 1995; (9) Maiolino et al. 1994.

tional velocity $v_{\text {rot }}$; velocity dispersion $\sigma_{v}$, fixed at $\sigma_{v}=10$ $\mathrm{km} \mathrm{s}^{-1}$, as is commonly observed in spiral galaxy disks (e.g., Shostak \& van der Kruit 1984); scale height $h$, fixed at 10 pc, self-consistent with the measured rotation velocities and disk radii; and, particularly for edge-on galaxies, inner and outer annular radii $r_{\text {in }}$ and $r_{\text {out }}$. The scale height $h$ for NGC 4151 was fixed at $0.1 \mathrm{pc}$ because of the small radial scale of

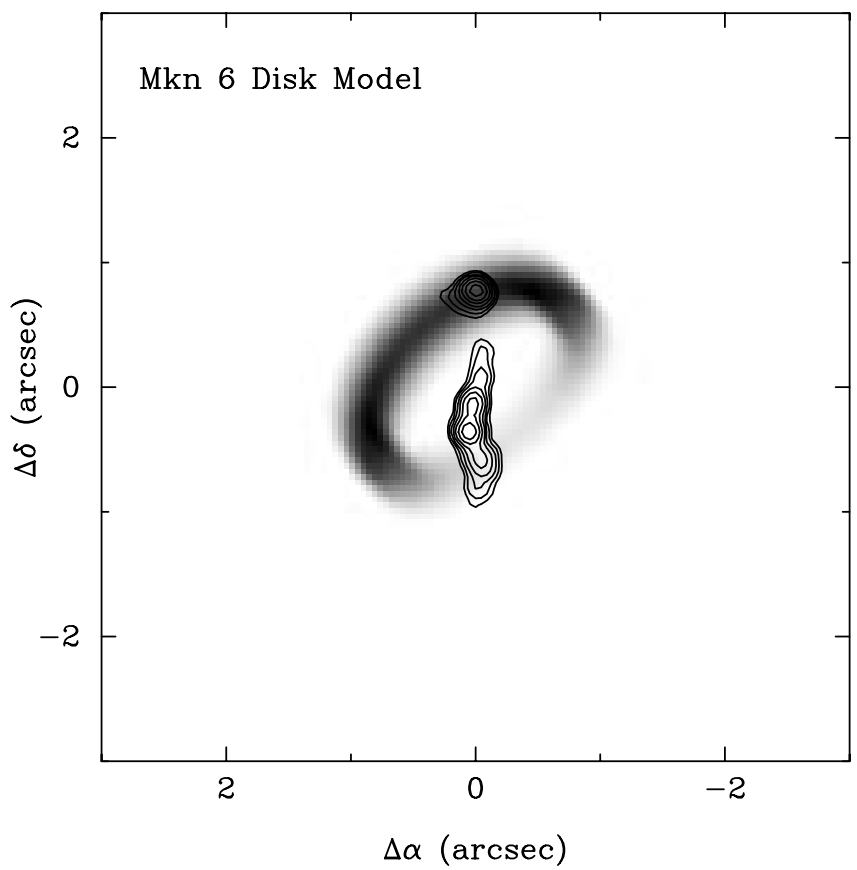

the absorbing disk. We also fitted the rotation curve as a power law $v \propto r^{\alpha}$, but, in every case, $\alpha \lesssim 0.01$, meaning an essentially flat rotation curve over the measured region.

Since the hypothesis to be tested is whether the $\mathrm{H}$ I absorption traces normal disk gas, the inclination $i$ and kinematic major axis $\phi$ were fixed to those values observed in the outer disk of the host galaxy in question. For NGC

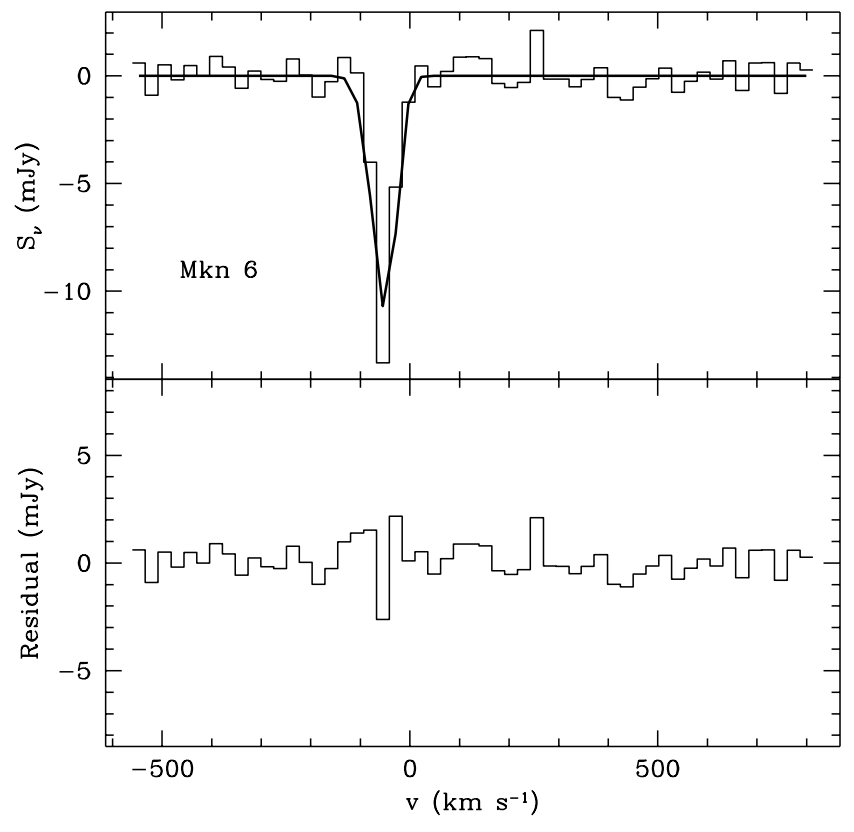

Fig. 15.-Kinematic model fit for the H I absorbing disk of Mrk 6. The data are MERLIN observations from Gallimore et al. (1998). Left panel: A schematic of the $\mathrm{H}$ I absorbing disk model. The disk has been shaded so that the far side appears lighter than the near side, as if it were viewed through an atmosphere; the effect is to mimic the appearance of a dust lane. The disk shading of the is lightened where there is significant background continuum, mainly to improve the visibility of the continuum contours. We erased the disk shading where the radio continuum structure lies foreground to the disk to illustrate the relative orientation of the disk and jet. The scale height of the disk has been exaggerated for presentation. The contours trace the $20 \mathrm{~cm}$ radio continuum image. Note that the outer radius of the H I disk is a lower limit set by the extent of the observed $\mathrm{H}$ I absorption. Right panel: Model spectra. The top panel shows the fit (thick line) against the $\mathrm{H}$ I spectrum (thin line), and the bottom panel shows the residuals. 

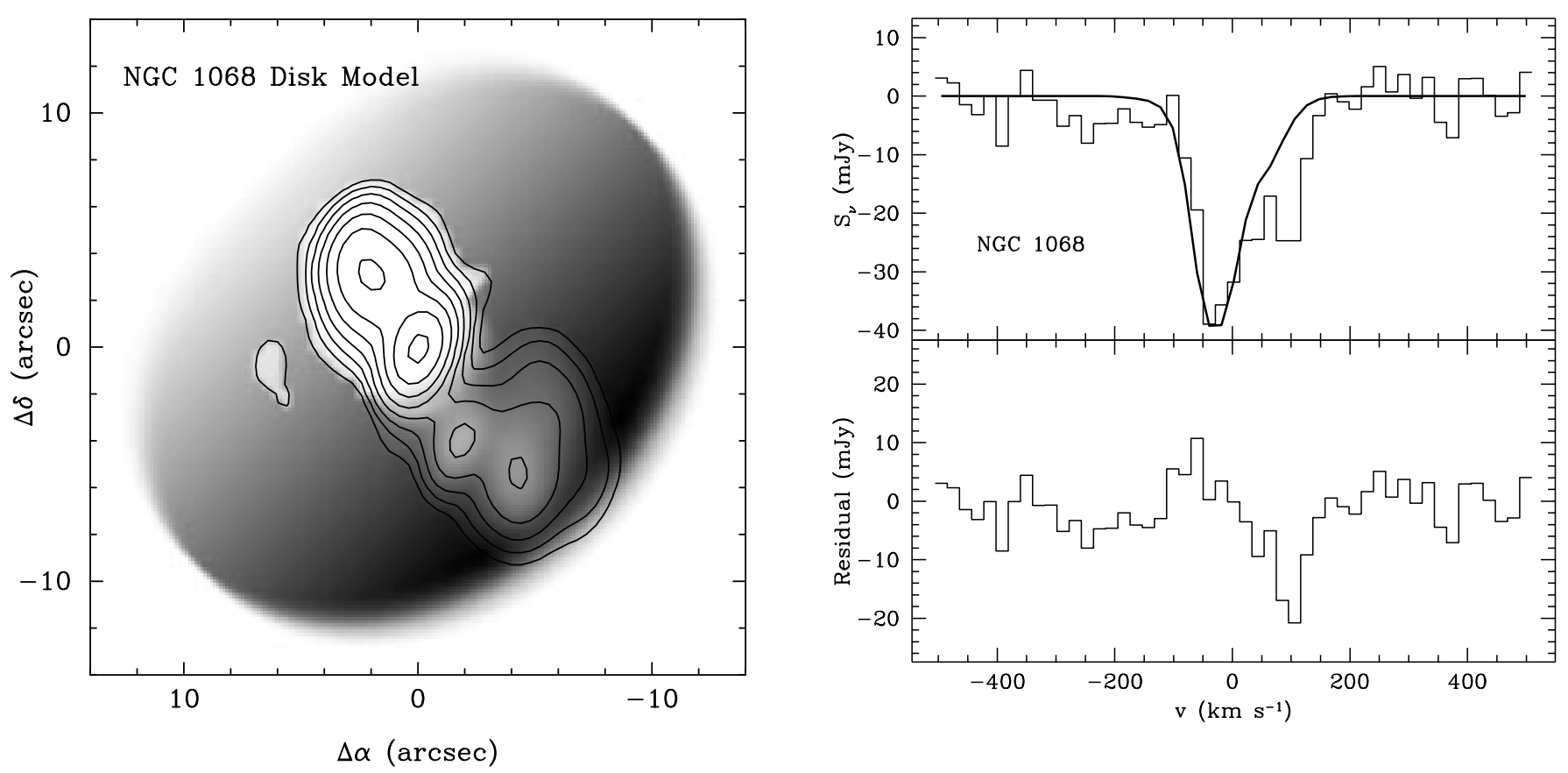

FIG. 16.-Kinematic model fit for the $\mathrm{H}$ I absorbing disk of NGC 1068. Left panel: A schematic of the $\mathrm{H}$ I absorbing disk model, following the convention of Fig. 15. The contours trace the $20 \mathrm{~cm}$ radio continuum image. The outer radius of the $\mathrm{H} \mathrm{I}$ disk is a lower limit set by the extent of the observed $\mathrm{H} \mathrm{I}$ absorption. Right panel: Model spectra. The top panel shows the fit (thick line) against the $\mathrm{H}$ I spectrum (thin line), and the bottom panel shows the residuals.

1068, we took into account the rotation of the kinematic axis by $45^{\circ}$ between the outer and inner galaxy. The apparent cause of this rotation is streaming in the central stellar bar (Gallimore et al. 1994). Lacking spatial information, except for NGC 1068, we further assumed that the absorbing $\mathrm{H}$ I uniformly fills the disk. In each case where the $\mathrm{H} \mathrm{I}$ absorption is displaced from the nucleus, we assumed that the near side of the disk lies on the same side of the nucleus

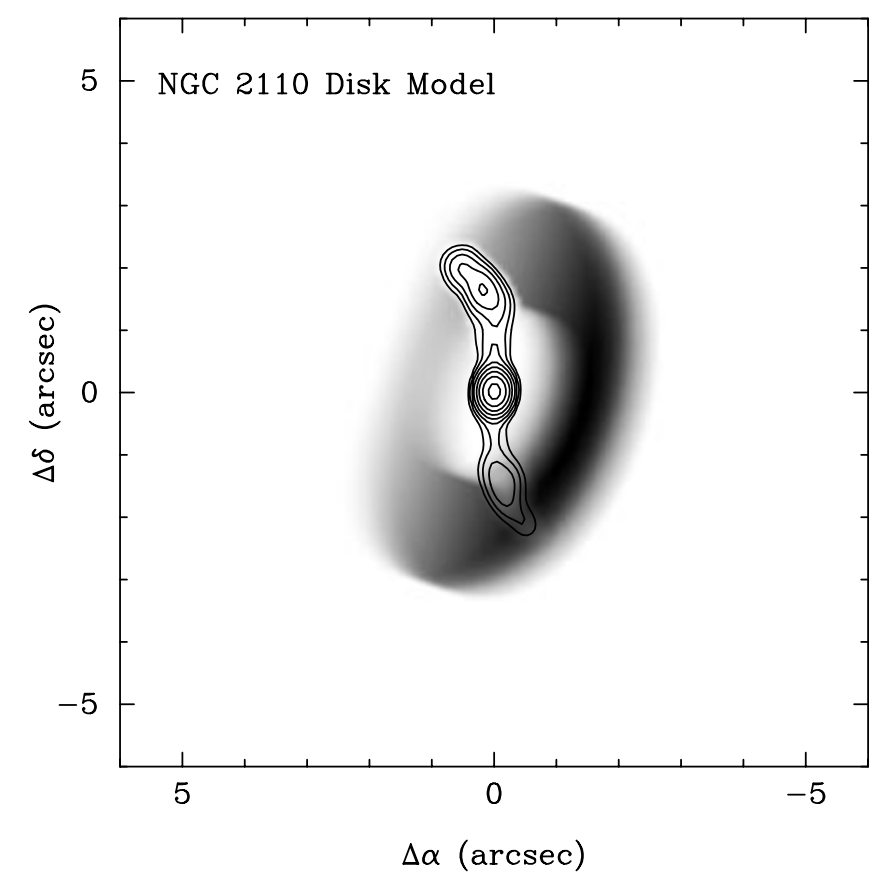

as the offset absorption. The (deprojected) extent of the observed $\mathrm{H}$ I absorption determines the inner and outer radii of the disk. For the edge-on galaxies in our survey, only the line profile constrains the inner and outer radii of the absorbing disk.

Having generated and properly oriented the model disk and background radio source, we then integrated the lineof-sight opacity $\tau(v)$ and computed an absorption spectrum

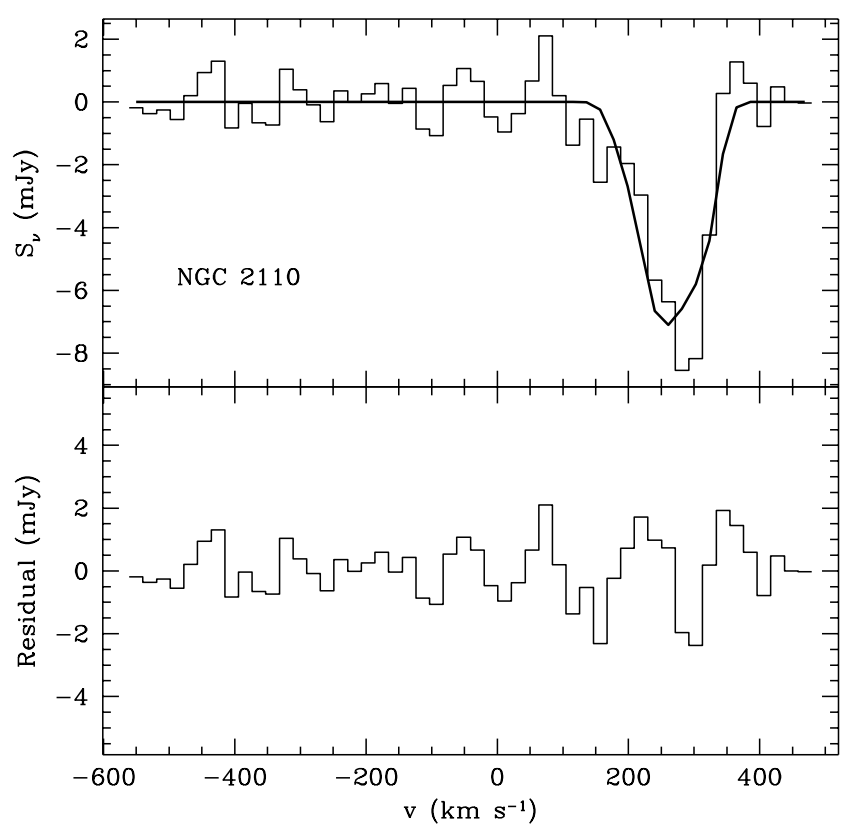

FIG. 17.-Kinematic model fit for the H I absorbing disk of NGC 2110. Left panel: Schematic view, following the convention of Fig. 15. The contours trace a $6 \mathrm{~cm}$ radio continuum image from Ulvestad \& Wilson (1984). We display the higher resolution VLA image to emphasize the H I absorbed radio structure. The outer radius of the $\mathrm{H}$ I disk is a lower limit set by the extent of the observed $\mathrm{H}$ I absorption. Right panel: Model spectra. The top panel shows the fit (thick line) against the $\mathrm{H}$ I spectrum (thin line), and the bottom panel shows the residuals. 

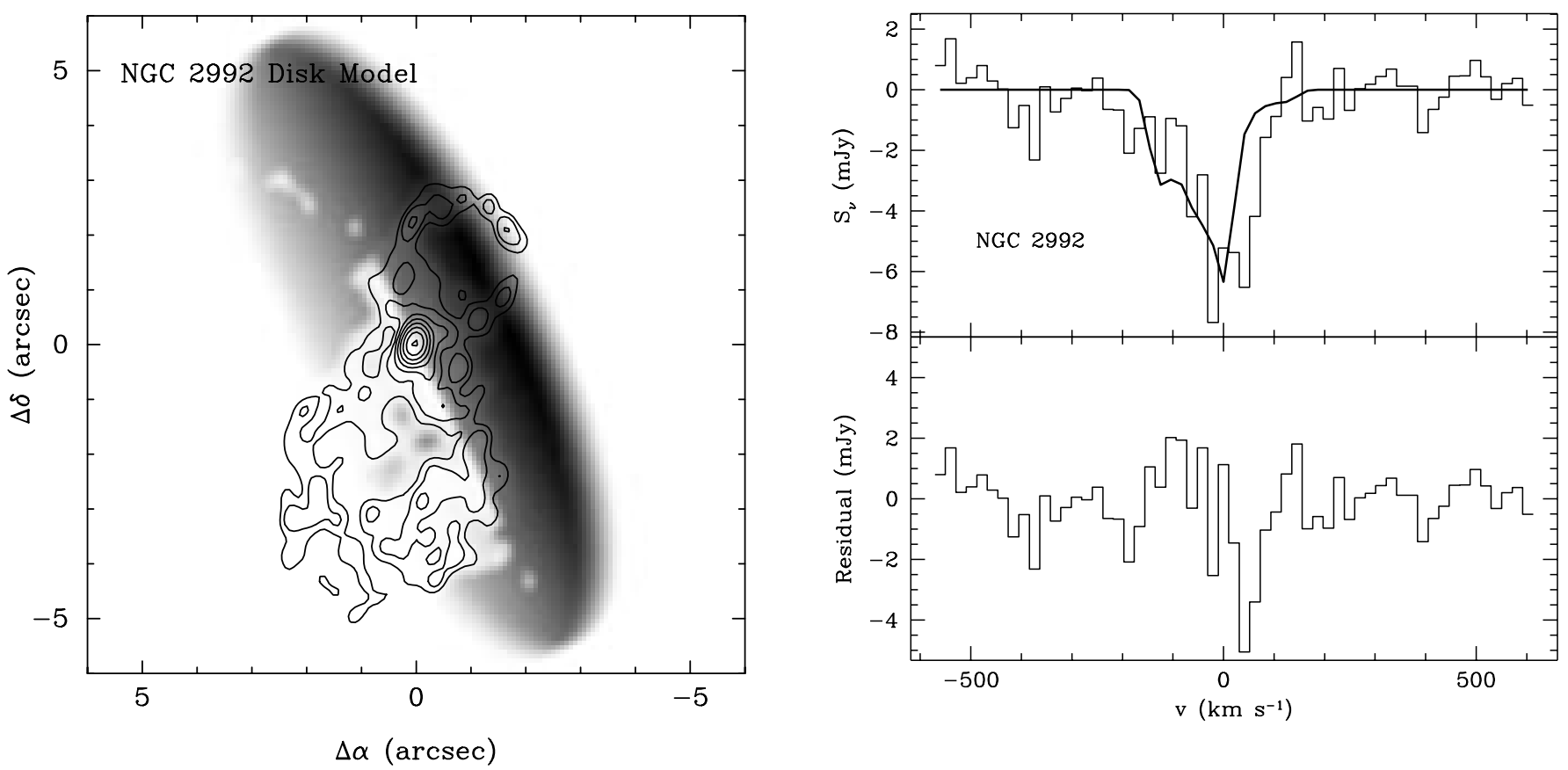

Fig. 18.-Kinematic model fit for the H I absorbing disk of NGC 2992. Left panel: Schematic view, following the convention of Fig. 15. The contours trace a $6 \mathrm{~cm}$ radio continuum image from Ulvestad \& Wilson (1984). The outer radius of the $\mathrm{H}$ I disk is a lower limit set by the extent of the observed $\mathrm{H}$ I absorption. Right panel: Model spectra. The top panel shows the fit (thick line) against the H I spectrum (thin line), and the bottom panel shows the residuals.

for each background CLEAN continuum component. The final spectrum was created by adding up all of the model absorption spectra. We fit the model spectrum against the observed $\mathrm{H}$ I absorption spectrum using a downhill simplex algorithm to minimize $\chi^{2}$ (chapter 10.4 of Press et al. 1992, and references therein). The best-fit model was then checked for self-consistency against the observed $\mathrm{H}$ I absorption morphology. We performed this check to ensure that the model disk would not obscure unabsorbed radio structure.

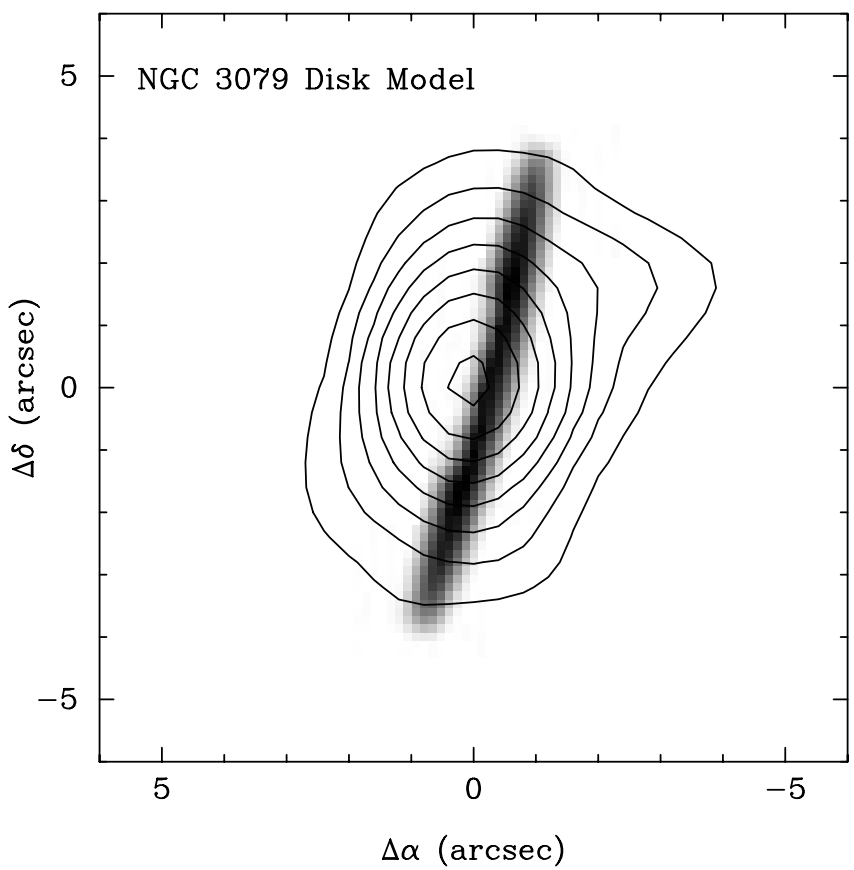

After convergence, we estimated standard errors using a Monte Carlo technique. We assumed that the best-fit model line profile was the correct profile and added random, Gaussian noise to the model spectrum to simulate an observed spectrum. The rms of the simulated noise was set equal to that of the residual spectrum (i.e., data minus model). We repeated the fitting procedure on a series of 10 simulated line profiles. The standard errors were derived from the deviations of parameters fitted to the simulated

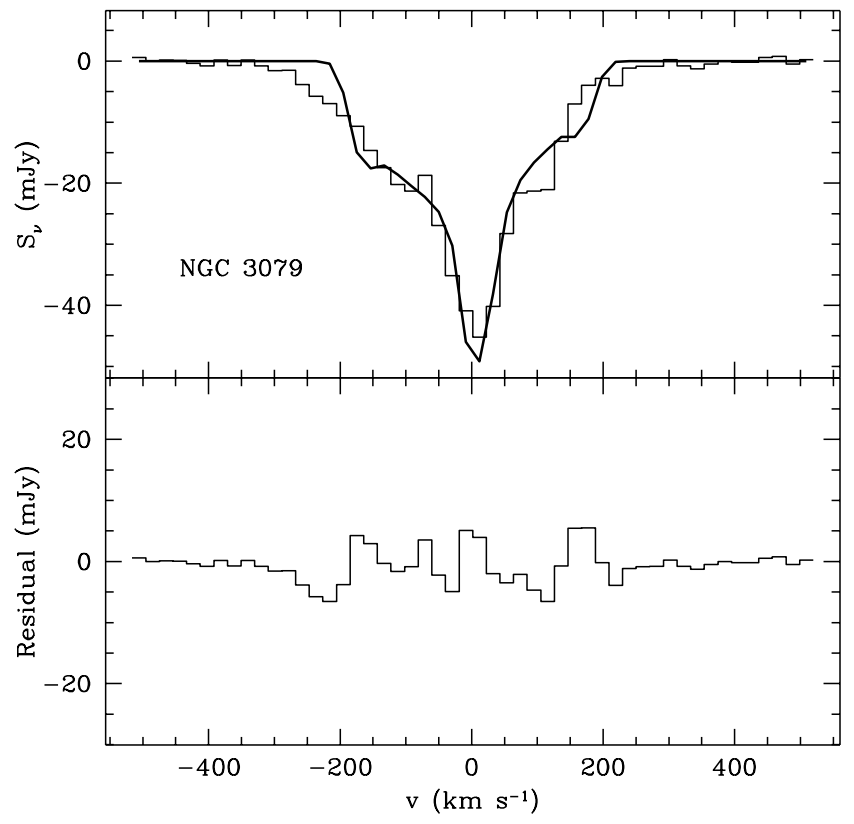

FIG. 19.-Kinematic model fit for the H I absorbing disk of NGC 3079. Left panel: Schematic view, following the convention of Fig. 15. The contours are $20 \mathrm{~cm}$ radio continuum. Right panel: Model spectra. The top panel shows the fit (thick line) against the $\mathrm{H}$ I spectrum (thin line), and the bottom panel shows the residuals. 

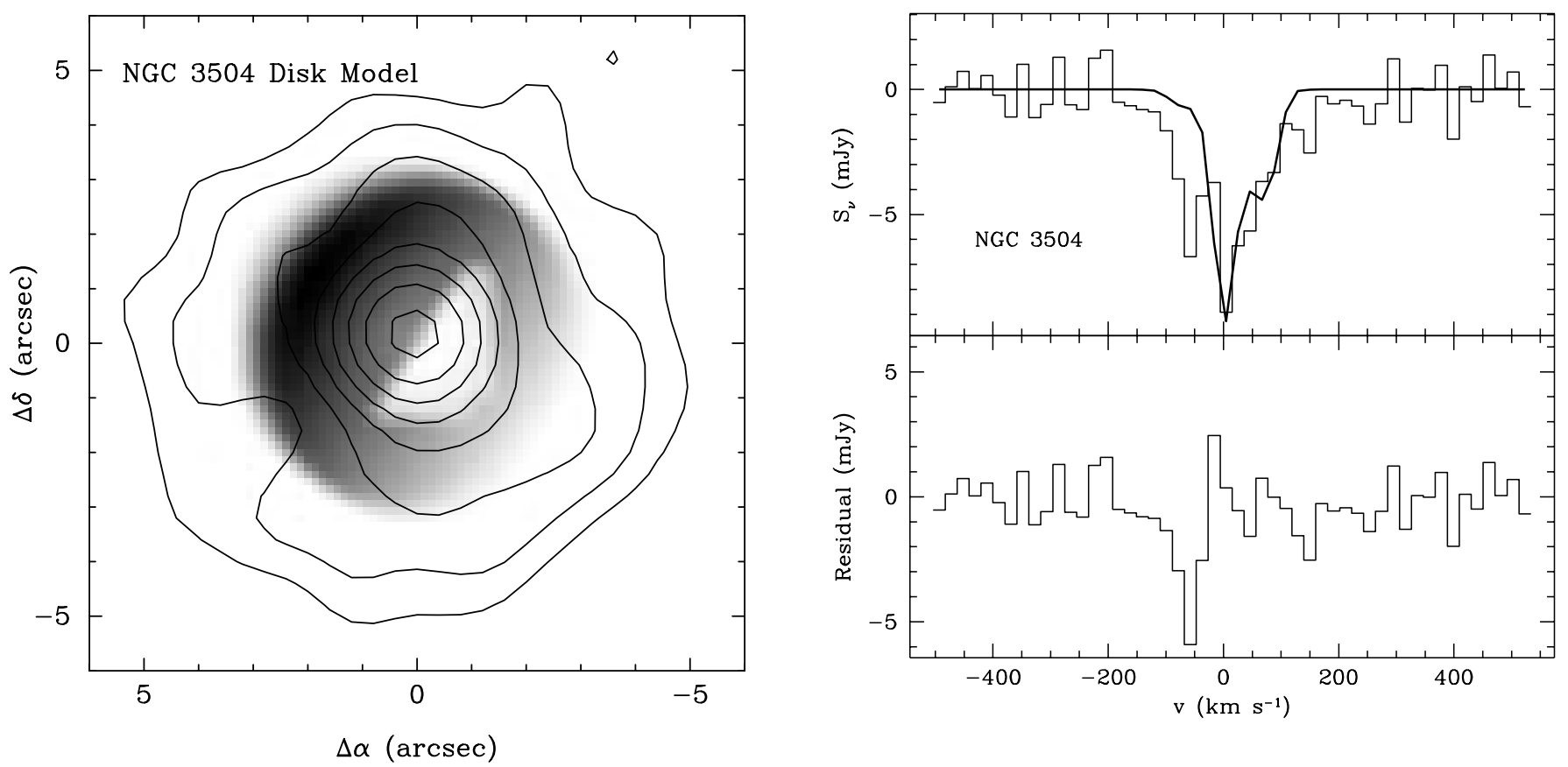

FIG. 20.-Kinematic model fit for the H I absorbing disk of NGC 3504. Left panel: Schematic view, following the convention of Fig. 15. The outer radius of the disk is poorly constrained, because the background continuum is so weak, and therefore offset $\mathrm{H}$ I absorption contributes little to the global profile. The contours are $20 \mathrm{~cm}$ radio continuum. Right panel: Model spectra. The top panel shows the fit (thick line) against the $\mathrm{H}$ I spectrum (thin line), and the bottom panel shows the residuals.

spectra. The failing of this technique is that it does not properly account for real absorption not present in the model and so may underestimate the real uncertainties where there are significant residuals. On the other hand, the measured standard errors appear to reflect the quality of the models and may not be far from their true values.

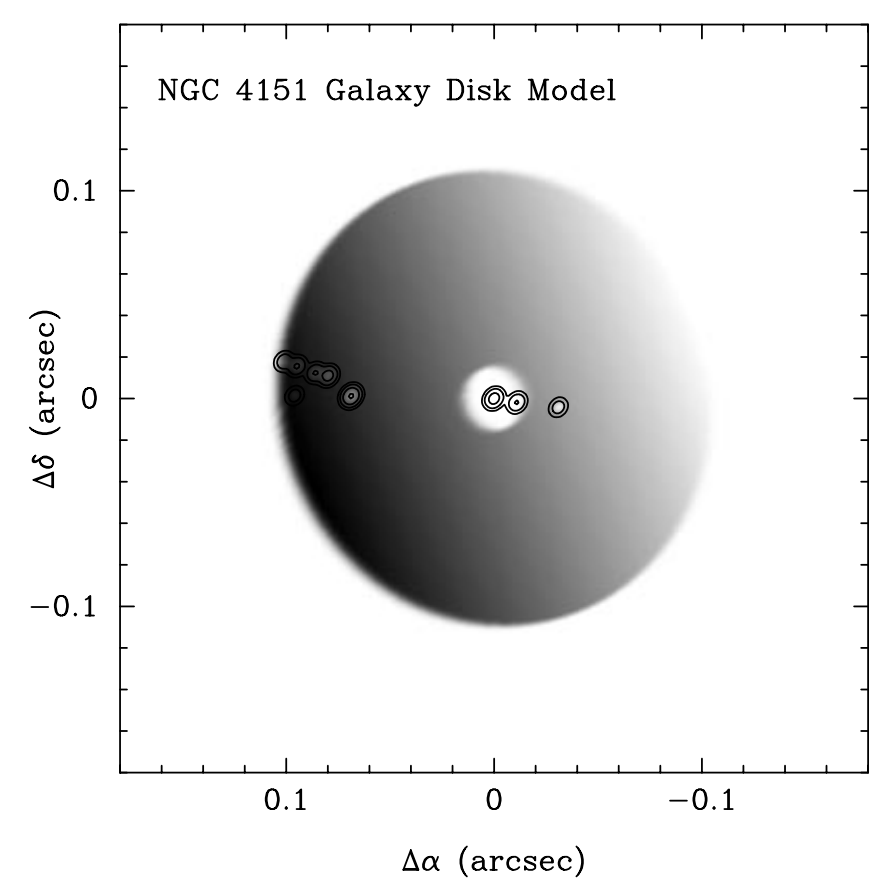

The models are not unique in the sense that one can always contrive a model in which all of the gas is falling into or flowing away from the background radio source. On the other hand, the structure of the background radio source leaves a "fingerprint" on the absorption profile that promotes the uniqueness of the fitted parameters of the disk

FIG. 21.-Kinematic model fit for the H I absorbing disk of NGC 4151. Left panel: Schematic view, following the convention of Fig. 15. The contours are $20 \mathrm{~cm}$ radio continuum modeled after the VLBI observations of Ulvestad et al. (1998). In this model, the disk is oriented with the same inclination and position angle as the outer galaxy disk. Right panel: The top panel shows the fit (thick line) against the H I spectrum (thin line), and the bottom panel shows the residuals. Note that this model requires a counterrotating disk. 

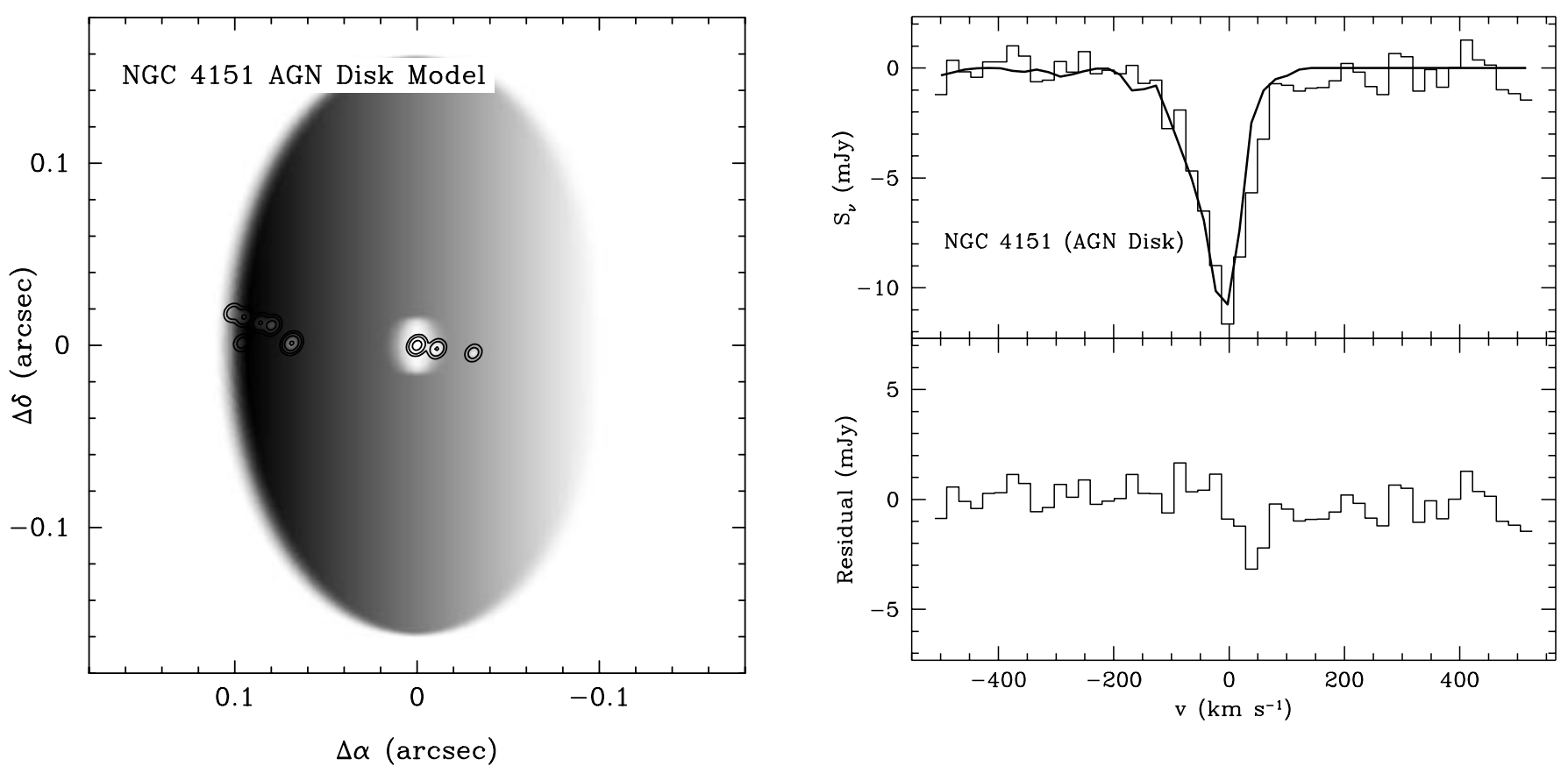

FIG. 22.-Kinematic model fit for the H I absorbing disk of NGC 4151. Left panel: Schematic view, following the convention of Fig. 15. The contours are $20 \mathrm{~cm}$ radio continuum modeled after the VLBI observations of Ulvestad et al. (1998). In this model, the disk is oriented with the same inclination and position angle as that inferred for an AGN disk (Mundell et al. 1995). Right panel: The top panel shows the fit (thick line) against the H i spectrum (thin line), and the bottom panel shows the residuals.

model. That is, the structure of the background source will preferentially enhance or suppress the contribution of certain velocities to the spatially integrated line profile. An important failing is that our models cannot fit for spatially varying opacity in the absorbing gas because plausible opacity variations, such as spiral arm structure, require too many parameters for the fits to be meaningful. Nevertheless,

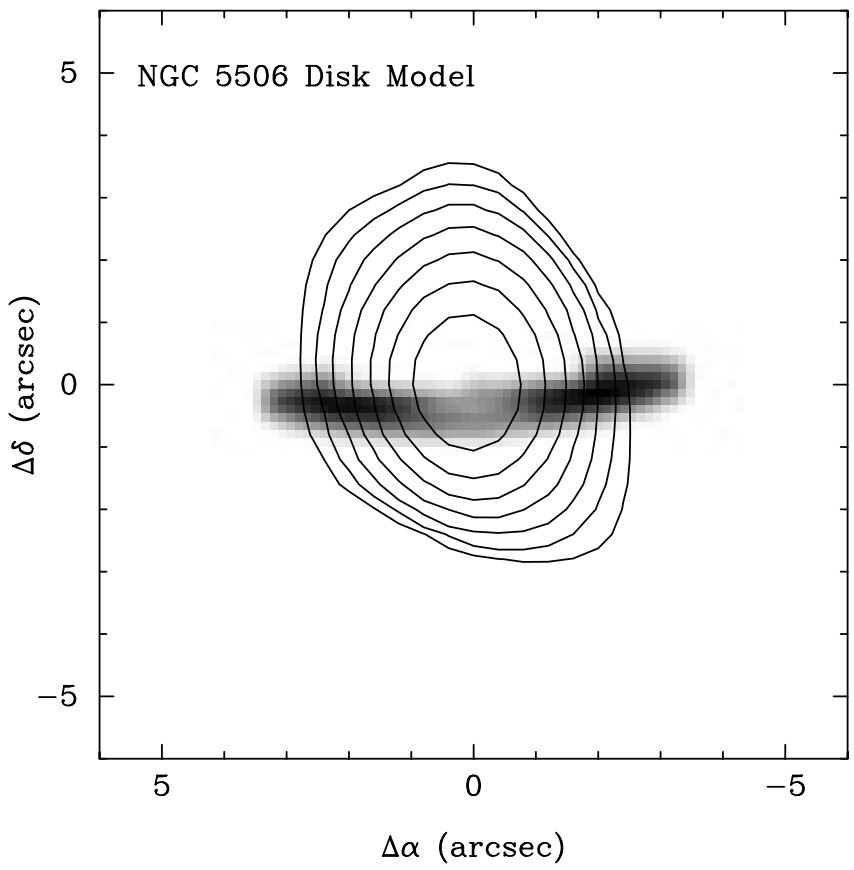

the uniform disk model produces a reasonable and illustrative fit for all of the observed line profiles.

One potential artifact of the fitting is a parsec-scale inner radius for the absorbing disk. This artifact results mainly from the need to fit the high-velocity wings of the absorption-line profile, since, for a given annular section of the disk at radius $r$ surrounding a radio source of size $r_{0}$, the

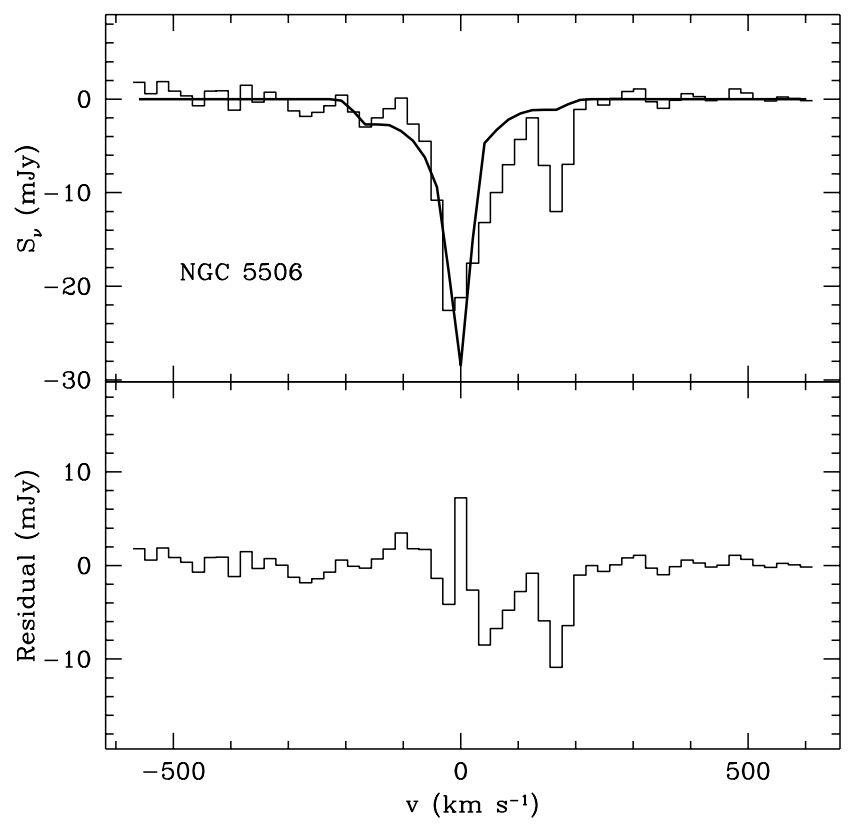

FIG. 23.-Kinematic model fit for the H I absorbing disk of NGC 5506. Left: Schematic view, following the convention of Fig. 15 . The contours are $20 \mathrm{~cm}$ radio continuum. Right panel: Model spectra. The top panel shows the fit (thick line) against the $\mathrm{H}$ I spectrum (thin line), and the bottom panel shows the residuals. 
velocity width is $\Delta v \sim v_{\text {rot }}\left\{r_{0} /\left[\left(r-r_{0}\right)^{2}+r_{0}^{2}\right]^{1 / 2}\right\}$. The need for a small inner radius might be relaxed by allowing larger velocity dispersions or streaming velocities. We chose to keep the dispersions fixed, since often the dispersions would grow unrealistically large in order to fit a discrete velocity component (e.g., NGC 1068, Fig. 16; and NGC 5506, Fig. 23). Fitted values for the disk inner radius should be viewed as a lower limit.

\subsection{Results}

With the exception of NGC 4151, which is described further below, the disk models generally describe the observed $\mathrm{H}$ I absorption profiles and locations very well. Poor values of $\chi^{2}$ can be traced generally to a discrete velocity component for which the simple disk model cannot account. Any discrete, residual components always arise within the velocity range traced by the model disk profile. We therefore suspect that unresolved opacity variations in the foreground disk may give rise to the discrete features in the line profile. For example, the spectrum of NGC 5506 shows a discrete redshifted component (Fig. 23) that might arise from a cold cloud in the main disk which happens to fall along the line of sight.

To demonstrate the validity of the galaxy disk model, we point to the peculiar absorption-line profile of NGC 2110 (Fig. 17). The absorption line is redshifted from systemic by nearly $300 \mathrm{~km} \mathrm{~s}^{-1}$, and the traditional interpretation would be infall toward the galaxy nucleus. However, the VLA images reveal that the absorbing gas is displaced from the nucleus along the kinematic major axis. Because of the special, chance alignment of the kinematic major axis and the radio jet, the northern lobe of the jet back-illuminates receding disk gas, and the result is a redshifted line profile. The uniform disk model reasonably reproduces the line profile shape, lending credence to this interpretation. The lack of a blueshifted absorption line against the opposing, southern radio lobe indicates that the jet points in front of the disk to the south.

NGC 4151 represents a special case, since the H I absorption probably traces gas within the inner few tens of parsecs of the nucleus. Unfortunately, the location of the nucleus in the radio reference frame is not known, since all of the compact continuum sources are steep spectrum (e.g., comparing Pedlar et al. 1993 and Carral, Turner, \& Ho 1990). Since the H I absorbed component (C4) is also free-free absorbed, and since the radio jet and NLR probably point toward us west of $\mathrm{C} 4$, the nucleus is probably near and west of C4 (Pedlar et al. 1998). We selected the central VLBI component $\mathrm{C} 4 \mathrm{~W}$ as the location of the nucleus after Mundell et al. (1995). Discussed below, this placement affects only the quantitative results of the model but does not alter the qualitative conclusions.

The radio jet of NGC 4151 does not align with the minor axis of the host galaxy, and so the AGN disk and galaxy disks are also misaligned. We therefore tried two disk models for NGC 4151, one with an orientation matching the host galaxy (Pedlar et al. 1993), and one matching the inferred properties of an AGN disk (Mundell et al. 1995). The AGN disk model fits the absorption spectrum better than the galaxy disk model. The reason is that the $\mathrm{H} \mathrm{I}$ absorption line centers very near the systemic velocity, and the AGN disk model places the $\mathrm{H}$ I absorbing gas along the disk minor axis. The galaxy disk model, on the other hand, predicts a slightly redshifted absorption component, since the absorption occurs toward the receding axis. As a result, the galaxy disk model requires counterrotation to fit the observed $\mathrm{H}$ I profiles. These conclusions hold so long as the radio jet originates at the kinematical center of the galaxy; the kinematical center may be placed anywhere along the radio jet and an AGN disk will always fit the absorption spectrum better than the galaxy disk model.

\subsection{Radial Streaming}

To look for evidence of infall that might fuel the AGN, we repeated the disk model fits allowing for a radial component to the gas velocity. The best-fit streaming velocities are listed in Table 7. The trivial, but unrealistic, solution would have been to set the rotational velocity to zero and assume purely radial motions. To avoid the trivial solution, we initialized the iterative fitting procedure with the parameters from the purely rotational model and allowed the circular and radial velocities to relax into a local minimum for $\chi^{2}$.

Although the error bars are usually large relative to the fitted values, all of the streaming velocities are radially inward toward the nucleus except for NGC 1068 and NGC 4151. The deprojected infall velocities are typically a few tens of $\mathrm{km} \mathrm{s}^{-1}$.

Looking at the exceptions, the $\mathrm{H}$ I absorbed region of NGC 1068 traces gas mainly on the trailing side of the central stellar bar Gallimore et al. 1994, over which the mean radial velocity of gas is expected to be away from the nucleus (e.g., Roberts, Huntley, \& van Albada 1979; Sanders \& Tubbs 1980). Although the streaming velocities in NGC 1068 are very uncertain, the implied outward streaming is consistent with the predicted bar streaming. It is all but impossible to interpret the outflowing gas motions in NGC 4151 since the absorption is not yet resolved against the VLBI structure.

An important caveat is the main failing of these disk models; namely, we cannot recreate the distribution of absorbing gas as a function of galactic coordinates. A localized enhancement of foreground column covering a finite range of radial velocities can mimic enhanced velocity dispersion or streaming velocities. Three-dimensional $(\alpha, \delta$, and $z$ ) models of the spectral line cubes would add enough information to allow at least a crude treatment on the spatial distribution of $\mathrm{H}_{\mathrm{I}}$ absorbing gas, but the present data generally lack the spatial resolution for threedimensional fits to be effective. The fitted streaming velocities can be viewed as upper limits to the true radial motions on $\sim 100$ pc scales.

Accepting this caveat, the upper limits on the massdeposition rates constrain the luminosity that can be powered by the $\mathrm{H}$ I disks. One concern, for example, is whether the upper limits on the infall rates might be insufficient to power the central engine. We have included in Table 7 the mass infall rates estimated by $\dot{M} \simeq$ $M_{\mathrm{HI}} v_{\text {rad }} / r_{\text {out }}$; typical values are a few times $0.1 M_{\odot} \mathrm{yr}^{-1}$. Among the $\mathrm{H}$ I detections presented here, the mean infall rate is $0.18 M_{\odot} \mathrm{yr}^{-1}$. This rate can support a nuclear luminosity of $L \simeq 10^{45}(\epsilon / 0.1) \mathrm{ergs} \mathrm{s}^{-1}$, where $\epsilon$ is the efficiency factor for mass-energy conversion in the accretion disk. This luminosity defines the traditional Seyfert/QSO luminosity boundary, and so the inferred mass-deposition rates are comparable to that required to fuel a Seyfert nucleus. We caution that this result does not constitute proof of infalling gas on $100 \mathrm{pc}$ scales. On the other hand, we may 
TABLE 7

STREAMING Velocity Models

\begin{tabular}{cccrr}
\hline \hline $\begin{array}{c}\text { Source } \\
(1)\end{array}$ & $\begin{array}{c}v_{\text {rad }} \\
\left(\mathrm{km} \mathrm{s}^{-1}\right) \\
(2)\end{array}$ & $\begin{array}{c}\dot{M} \\
\left(M_{\odot} \mathrm{yr}^{-1}\right)\end{array}$ & $\begin{array}{c}\chi^{2} \\
(3)\end{array}$ & \multicolumn{1}{c}{$\begin{array}{c}\Delta \chi^{2} \\
(5)\end{array}$} \\
\hline Mrk $6 \ldots \ldots \ldots$. & $<59.0$ & $<0.57$ & 95.4 & 48.4 \\
NGC $1068 \ldots \ldots$. & $-46.6 \pm 11.2$ & $-0.90 \pm 0.21$ & 36.8 & 27.7 \\
NGC $2110 \ldots \ldots$. & $47.3 \pm 11.7$ & $1.16 \pm 0.22$ & 42.4 & 14.6 \\
NGC $299 \ldots \ldots \ldots$ & $37.6 \pm 5.4$ & $0.67 \pm 0.09$ & 131.8 & 54.1 \\
NGC $3079 \ldots \ldots$. & $16.6 \pm 0.6$ & $0.18 \pm 0.01$ & 927.1 & 161.9 \\
NGC $3504 \ldots \ldots$. & $9.6 \pm 19.4$ & $0.18 \pm 0.36$ & 50.5 & 0.3 \\
NGC $4151^{\mathrm{a}} \ldots \ldots$. & $-0.32 \pm 0.04$ & $-182.4 \pm 22.5$ & 48.5 & 109.3 \\
NGC $4151^{\mathrm{b}} \ldots \ldots$. & $-0.07 \pm 0.01$ & $-24.8 \pm 3.8$ & 46.1 & 20.5 \\
NGC $5506 \ldots \ldots$. & $19.3 \pm 0.9$ & $0.05 \pm 0.01$ & 715.8 & 247.4 \\
\hline
\end{tabular}

NoTES.-Streaming velocities based on disk model fitting to the $\mathrm{H}$ I absorption profiles and locations. Positive velocities imply motion toward the center of the galaxy. All models also include a rotational component, but the fitted values are not significantly different from those listed in Table 6 . The exception is Mrk 6, for which the fitted rotational velocity drops to $\sim 40 \mathrm{~km} \mathrm{~s}^{-1}$; the fitted value therefore places a limit appropriate for gas dominated by radial motion. Col. (1): Source ID; col. (2): streaming velocity; col. (3): corresponding mass infall rate; col. (4): $\chi^{2}$; col. (5): $\Delta \chi^{2}$, the improvement in $\chi^{2}$ relative to the purely rotational model (Table 6).

${ }^{a}$ The inclination and position angle are fixed to those of the outer galaxy disk.

b The inclination and position angle are fixed to those predicted for an AGN disk (i.e., perpendicular to the radio jet).

conclude that the kinematics of the $\mathrm{H}$ I absorption are compatible with the standard model for AGNs, namely, that the active nucleus is fueled by gas removed from the host galaxy disk.

We should point out that the nature of the $\mathrm{H}$ I absorption in active spirals appears to be very different from the $\mathrm{H} \mathrm{I}$ absorption in active ellipticals. Apart from a substantially lower detection rate, van Gorkom et al. (1989) found that the $\mathrm{H}$ I absorption lines were never blueshifted, indicating either tangential motion or radial infall toward the central radio source. In contrast, we find that the kinematics of $\mathrm{H} \mathrm{I}$ absorption in Seyfert galaxies appear to be dominated by rotation, with a weak infall component superimposed.

\subsection{Stability Consideration}

Rotating gaseous disks are unstable to internal collapse and star formation where the disk exceeds a critical surface density $\Sigma_{c}$. Neglecting, for now, the contribution of molecular gas and ionized gas to the local surface density, the ratio of the neutral hydrogen surface density $\Sigma_{\mathrm{H}_{\mathrm{I}}}$ to the critical density is

$$
\frac{\Sigma_{\mathrm{H}}}{\Sigma_{c}} \simeq \frac{0.028 N_{21} v_{\mathrm{rot}}}{r_{\mathrm{pc}}},
$$

where $N_{21}$ is the neutral hydrogen column density in units $10^{21} \mathrm{~cm}^{-2}, v_{\text {rot }}$ is the rotational velocity in $\mathrm{km} \mathrm{s}^{-1}$, and $r_{\mathrm{pc}}$ is the radius in parsecs (based on Toomre 1964; Cowie 1981; Kennicutt 1989). We measure $\Sigma_{\mathrm{H}_{\mathrm{I}}} / \Sigma_{c} \sim 0.01-0.1$ for the model $\mathrm{H}_{\mathrm{I}}$ absorbing disks, based on the columns reported in Table 6 . The extreme values are $\lesssim 0.001$ for NGC 4151 (AGN disk) and NGC 5506 and 0.45 for NGC 4151 (Galaxy Disk).

The $\mathrm{H}$ I surface densities generally fall well below the critical density, but the surface density of molecular and ionized gas might still push the disks to instability. In the limit where atomic gas is the minority phase, the stability criterion becomes

$$
\frac{\Sigma_{\mathrm{H}}}{\Sigma_{\mathrm{H}_{\mathrm{I}}}} \lesssim\left(\frac{\Sigma_{\mathrm{HI}_{\mathrm{I}}}}{\Sigma_{c}}\right)^{-1} .
$$

That is, for these disks to be unstable to star formation, the molecular and ionized gas masses would have to be $10-100$ times the atomic gas mass over the inner kiloparsec.

In global averages, the surface densities of atomic and molecular gas in spiral galaxies are similar to within factors of a few (e.g., Kennicutt 1990, and references therein). On the other hand, gas in the centers of spiral galaxies tends to be predominantly molecular (e.g., Jackson et al. 1996; Helfer \& Blitz 1997). Taking NGC 1068 as an example, Helfer \& Blitz (1995) estimate a molecular gas column $N_{\mathrm{H} 2} \sim 5 \times 10^{22} \mathrm{~cm}^{-2}$ near the region we detect $\mathrm{H} \mathrm{I}$ absorption. The molecular surface density exceeds the $\mathrm{H} \mathrm{I}$ surface density by a factor of $\gtrsim 20$, pushing $\Sigma / \Sigma_{c} \gtrsim 1$. On the other hand, the rotation curve remains poorly constrained on this scale; a radial gradient of the rotation velocity would raise $\Sigma_{c}$ and stabilize the disk.

In NGC 1068, at least, $\mathrm{H}$ I is the probably the minority phase within the inner 100 pc. If other Seyfert galaxies are similarly rich in molecular gas, the disks traced by $\mathrm{H} \mathrm{I}$ absorption disks may be unstable to star formation. Unfortunately, we are unable to test this suggestion with the present data. Current and future millimeter surveys, such as that currently taking place at the Berkeley-Illinois-Maryland Association (BIMA) telescope (e.g., Helfer et al. 1998), may better address the stability issue in other Seyfert galaxies.

\section{IMPLICATIONS FOR UNIFYING SCHEMES}

Perhaps the most surprising result of this survey is the lack of $\mathrm{H}$ I absorption toward the AGN in Seyfert nuclei, particularly those known to harbor heavily obscured central engines. The trivial explanation is that the central 
X-ray sources are radio silent. However, the well-known hidden Seyfert 1 nuclei are also the most luminous Seyfert galaxies at radio wavelengths (Moran et al. 1992), and it seems unlikely that the most luminous radio Seyfert galaxies contain radio-silent AGNs. Another possibility is that the obscuring disks are entirely molecular, or, alternatively, that our sight line crosses mainly through an ionized region of the disk. The line-of-sight X-ray columns commonly exceed $10^{21} \mathrm{~cm}^{-2}$ (Table 5). Regardless of the viewing geometry, at some distance from the central ionizing source the obscuring material will become largely neutral. Photodissociation models predict a substantial atomic column detectable in $21 \mathrm{~cm}$ absorption, even if most of the material is molecular; for example, in the presence of an Seyfert nucleus, dissociative excitations can liberate atomic gas within molecular clouds located out to distances of hundreds of parsecs (Maloney et al. 1996).

We next consider three ways in which $\mathrm{H}$ I absorption from AGN obscuring disks might be suppressed, namely, kinematical line broadening, free-free absorption of nearnuclear radio continuum, and excitation effects for the 21 $\mathrm{cm}$ line. In each case the implication is that, ironically, the obscuring disk must be small, probably parsecs in scale, in order to suppress $\mathrm{H}_{\mathrm{I}}$ absorption from the obscuring material. On the other hand, $\gtrsim 100 \mathrm{pc}$ obscuring disks would not suffer these suppressing effects, and the prediction would have been strong $\mathrm{H}$ I absorption, contrary to the observations.

\subsection{Kinematical Line Broadening}

For a given mass of obscuring $\mathrm{H} \mathrm{I}$, the absorption-line strength is inversely proportional to the velocity width of the line. In the environment of a compact mass, the absorption-line width should vary inversely with the (inner) radius from the $A G N$, and lines arising from rapidly moving gas near an AGN may be broadened below detectability. Without loss of generality, we consider a model where the ensemble of obscuring gas clouds is supported by random motion against gravitational collapse. Our $21 \mathrm{~cm}$ absorption experiment would be sensitive to columns:

$$
N_{\mathrm{H} \mathrm{I}} \approx 3 \times 10^{21} r_{\mathrm{pc}}^{-1 / 2} M_{7}^{1 / 2}\left(\frac{\sigma_{\mathrm{mJy}}}{C_{100}}\right)\left(\frac{T_{\mathrm{spin}}}{8000 \mathrm{~K}}\right) \mathrm{cm}^{-2},
$$

where $r_{\mathrm{pc}}$ is the distance from the AGN, $M_{7}$ is the mass of the black hole in units $10^{7} M_{\odot}, \sigma_{\mathrm{mJy}}$ is the channel map sensitivity in units of $\mathrm{mJy}$, and $C_{100}$ is the background continuum strength in units of $100 \mathrm{mJy}$ (e.g., as is appropriate for Mrk 348). We have also assumed that the local spin temperature is enhanced to $\sim 8000 \mathrm{~K}$, as may obtain in the warm atomic layer near an AGN (Maloney et al. 1996). We conclude that the absorption experiment remains sensitive to column densities that may be typical for obscured Seyfert 1 nuclei, $N_{\mathrm{H}} \gtrsim 10^{23} \mathrm{~cm}^{-2}$, in the presence of kinematical line broadening. The obscuring gas would have to be within $\sim 1 C_{100}^{-2} \mathrm{mpc}$ of the AGN to suppress $\mathrm{H}$ I absorption below detectability.

\subsection{Free-Free Absorption}

The hypothesis in this case is that, as has been verified for NGC 1068 (e.g., Gallimore, Baum, \& O’Dea 1996, 1997; Roy et al. 1998), the nuclear continuum source has been attenuated by free-free absorption. Therefore, $\mathrm{H}_{\mathrm{I}}$ in the neutral region of the obscuring torus lacks a background source on the smallest scales, and $\mathrm{H}$ I absorption would not be detected. In this scenario, nuclear radio continuum does not trace emission from the AGN proper but instead from compact sources in the central, unresolved region of the radio jet. This is certainly the case for NGC 1068 (e.g., Gallimore et al. 1996b) and Mrk 231 (Carilli et al. 1997), for example.

Further motivating this hypothesis, the X-ray photoionization models of Krolik \& Lepp (1989) and Neufeld, Maloney, \& Conger (1994) have demonstrated that X-ray heated plasma within a parsec-scale obscuring torus can be optically thick to free-free absorption down to $\sim 1 \mathrm{~cm}$ wavelength. Observationally, VLBI surveys are revealing free-free absorbed radio sources in other Seyfert nuclei (e.g., Ulvestad et al. 1999). Pedlar et al. (1998) find evidence for low-frequency free-free absorption toward the nucleus of NGC 4151. The paucity of obvious radio cores in Seyfert nuclei (e.g., Wilson 1991), even relatively radio-bright Seyfert nuclei, also argues for free-free suppression of the Seyfert radio nuclei.

We next demonstrate that the condition of free-free absorption places limits on the scale of the free-free obscuring plasma, which in turn limits the inner radius for neutral gas in the obscuring torus. For a given free-free opacity (i.e., emission measure), the free-free radio continuum flux and hydrogen recombination-line flux scale with the projected surface area of the free-free absorbing plasma. Looking first at radio continuum emission from the nucleus, the constraint on the nuclear free-free radio surface brightness gives

$$
r \lesssim 18 D_{20} \sigma_{\mathrm{mJy}}^{1 / 2} T_{4}^{-1 / 2} C_{f}^{-1 / 2} \mathrm{pc},
$$

where $D_{20}$ is the distance in units of $20 \mathrm{Mpc}, \sigma_{\mathrm{mJy}}$ is the channel map sensitivity in $\mathrm{mJy}, T_{4}$ is the electron temperature in units $10^{4} \mathrm{~K}$, and $C_{f}$ is the covering fraction of the obscuring medium. This limit also assumes that the brightness temperature is equal to the electron temperature, appropriate for optically thick thermal plasma.

Since, at $1^{\prime \prime}$ resolution, any free-free emission from the obscuring torus would probably be confused with synchrotron emission from the jet, we are limited not by the continuum sensitivity but rather the $\mathrm{H}$ I absorption-line sensitivity. (As new VLBI maps of Seyfert galaxies are published, continuum constraints on putative free-free absorbing regions may tighten the constraints argued here). X-ray absorbing columns of $10^{23} \mathrm{~cm}^{-2}$ (e.g., Table 5) produce an $\mathrm{H}_{\mathrm{I}}$ opacity of $\tau_{\mathrm{H}_{\mathrm{I}}} \sim 20 N_{23} /\left(T_{100} \Delta v_{100}\right)$, where $T_{100}=T_{S} / 100 \mathrm{~K}$ and $\Delta v_{100}$ is the line width in units of 100 $\mathrm{km} \mathrm{s} \mathrm{s}^{-1}$. A nuclear free-free continuum source would be completely attenuated over the $21 \mathrm{~cm}$ line profile, and the limit on the AGN radio continuum flux is therefore the detection limit for a single channel, or roughly $3 \mathrm{mJy}$ for this experiment. $C_{f} \sim 50 \%$ based on the ratio of Seyfert 1 to Seyfert 2 galaxies (Lawrence 1991). Applying these values, the obscuring plasma must be smaller than $r \sim 44 D_{20} \mathrm{pc}$, or else the background radio continuum provided by thermal free-free emission would produce an observable $\mathrm{H}$ I absorption line.

We next consider the hydrogen recombination-line luminosity of the free-free obscuring region. We choose to consider hydrogen recombination lines mainly for ease of computation; consideration of forbidden-line emission would require a photoionization model, which is beyond the scope of this work. Without loss of generality, we consider only $\mathrm{H} \alpha$ line emission. Since, according to unifying 
schemes, the ionized region of the torus would not be obscured by dust in Seyfert 1 galaxies, the spatially integrated $\mathrm{H} \alpha$ luminosities of the NLR provide an upper limit to the emission from the obscuring region. For Seyfert galaxies, the median NLR $\mathrm{H} \alpha$ luminosity is $L_{\mathrm{H} \alpha} \sim 10^{41}$ ergs $\mathrm{s}^{-1}$ (based on characteristic line ratios in Ferland \& Osterbrock 1986 and observed $\mathrm{H} \beta$ luminosities from Whittle 1992). Taking the usual assumption of case-B recombination, the size of the free-free obscuring region must be

$$
r \lesssim 28\left(L_{\mathrm{H} \alpha} / 10^{41}\right) C_{f}^{-1 / 2} \tau_{\mathrm{rad}}^{-1 / 2} T_{4}^{-0.675} \mathrm{pc},
$$

where $\tau_{\mathrm{ff}}$ is the free-free opacity at the $21 \mathrm{~cm}$ rest wavelength and we have used the usual radio approximation for the free-free opacity law from Mezger \& Henderson (1967).

If free-free suppression of the nuclear continuum source commonly occurs in Seyfert nuclei, as is at least suggested by this $\mathrm{H}$ I absorption experiment, the implication is that the obscuring torus cannot be much larger than a few tens of parsecs. Turning the argument around, we would not predict substantial free-free absorption from a $100 \mathrm{pc}$ scale obscuring torus; rather, we would have expected strong $\mathrm{H} \mathrm{I}$ absorption against a bright continuum source.

\subsection{Excitation Effects}

The third way to suppress $\mathrm{H}$ I absorption is to raise the spin temperature, dropping the opacity for a given foreground column. We have demonstrated that, for typical radio source luminosities, the $21 \mathrm{~cm}$ transition in Seyfert obscuring disks is likely to be thermalized, and $\mathrm{H}$ I absorption should be detected (§ 4.1). On the other hand, particularly luminous nuclear radio sources will drive the local spin temperature to the equivalent brightness temperature of the local $21 \mathrm{~cm}$ continuum radiation field (e.g., Bahcall \& Ekers 1969). The radiation field from a compact nuclear source will drop as $r^{-2}$, and so excitation effects will affect $21 \mathrm{~cm}$ absorption only if the obscuring gas is located near the central engine. Since the hypothesis is that we see the bright radio nucleus directly, this case can be viewed as the opposite extreme of the free-free absorption scenario presented above.

In particular, spin temperature effects may explain the lack of $\mathrm{H}$ I absorption toward Mrk 348. Mrk 348 has an unusually bright nuclear radio source, $S_{v} \approx 100-500 \mathrm{mJy}$ at $21 \mathrm{~cm}$. This source is variable on timescales of years, marking it as a likely location of the AGN (e.g., Neff \& de Bruyn 1983; Unger et al. 1984). Assuming the source is much smaller than $1 \mathrm{pc}$, the $21 \mathrm{~cm}$ continuum temperature as a function of radius is

$$
T_{R} \approx 2.4 \times 10^{10} r_{\mathrm{pc}}^{-2} \mathrm{~K} .
$$

Applying the formalism of Bahcall \& Ekers (1969), the 21 $\mathrm{cm}$ radiation field will dominate the effects of particle collisions at radii

$$
r \lesssim 5 S_{100}^{1 / 2} n_{5}^{-1 / 2} D_{60} T_{100}^{0.1} \mathrm{pc},
$$

where $S_{100}$ is the continuum source brightness in units of $100 \mathrm{mJy}, n_{5}$ is the gas density in units $10^{5} \mathrm{~cm}^{-3}, D_{60}$ is the distance to the source in units of $60 \mathrm{Mpc}$, and $T_{100}$ is the kinetic temperature in units of $100 \mathrm{~K}$. The normalizations are specific to Mrk 348, but the result can be scaled and applied to any compact radio source. We estimate that the spin temperature might be as high as $\sim 10^{6} \mathrm{~K}$ at a radius of
$1 \mathrm{pc}$, and, as a result, $21 \mathrm{~cm}$ absorption opacity would be suppressed by a factor of $10^{4} T_{100}^{-1}$ !

\subsection{Conclusions}

Sanders et al. (1989) originally proposed that the obscuring medium in Seyfert galaxies might span scales much larger than the conventional torus model. More recently, Malkan, Gorjian, \& Tam (1998) reported that optical images of Seyfert 2 galaxies are more likely to show $\sim 100$ pc dust absorption features than optical images of Seyfert 1 galaxies. They argued that these galactic dust lanes are primarily responsible for hiding the central engines and BLRs in Seyfert 2 nuclei. The characteristic scale of the obscuring medium in this galactic dust model is $\gtrsim 100 \mathrm{pc}$. To some degree galaxy disks must obscure Seyfert nuclei, since Seyfert nuclei are rarely found in edge-on disk galaxies (Keel 1980; Simcoe et al. 1997).

To explain the surprising lack of $\mathrm{H}$ I absorption toward known, hidden Seyfert 1 nuclei requires, ironically, obscuring disks on $10 \mathrm{pc}$ scales or less. Turning the arguments around, we would expect that kinematical line broadening, free-free absorption, and excitation effects would have little effect on $21 \mathrm{~cm}$ absorption from gas on $100 \mathrm{pc}$ scales. Therefore, the galactic dust model predicts pronounced $\mathrm{H}$ I absorption toward radio-bright Seyfert nuclei. We find instead that the dust lanes observed by Malkan, Gorjian, \& Tam (1998) are probably the source of the off-nuclear H I absorption reported here. We conclude that the inner scale of the obscuring medium in hidden Seyfert 1 galaxies must be less than a few tens of parsecs, in better agreement with the classical parsec-scale torus scenario.

It is all but certain that free-free suppression of the nuclear continuum source has precluded the detection of 21 $\mathrm{cm}$ absorption toward NGC 1068. It seems very likely that excitation effects have suppressed $21 \mathrm{~cm}$ absorption in Mrk 348. The case of Mrk 3 is more difficult to address. Kukula et al. (1999) identify a compact, flat spectrum VLBI source as the nucleus. The source is weak, only $9 \mathrm{mJy}$ at $18 \mathrm{~cm}$, and so excitation effects will be important only on subparsec scales (scaling from eq. [8], above). On the other hand, this source might be intrinsically steep-spectrum but free-free absorbed at $18 \mathrm{~cm}$, in which case it need not be identified as the nucleus. Additional spectral information is needed to interpret the nuclear radio source and the nondetection of $21 \mathrm{~cm}$ absorption. Otherwise, we have demonstrated that, for plausible conditions in Seyfert nuclei, either free-free absorption or excitation effects would suppress $21 \mathrm{~cm}$ absorption from parsec-scale obscuring disks. These effects might naturally explain the tendency for $21 \mathrm{~cm}$ absorption to avoid the nuclei of obscured Seyfert galaxies.

\section{IMPLICATIONS FOR AGN FUELING}

One of the long-standing problems of AGN physics is the nature of the fuel source for the central engine. Based, for example, on source counts or the sizes of radio jets, the lifetime of an AGN is expected to exceed $\sim 10^{8} \mathrm{yr}$ (e.g., as reviewed by Peterson 1997). Supporting a typical Seyfert luminosity of $10^{44} \mathrm{ergs} \mathrm{s}^{-1}$ requires a fueling rate of about $0.02(\epsilon / 0.1) M_{\odot} \mathrm{yr}^{-1}$. Integrated over its lifetime, the central engine needs a total mass of $\sim 2 \times 10^{6}(\epsilon / 0.1) M_{\odot}$. This mass is comparable to the central $\mathrm{H}$ I masses derived in this absorption experiment (Table 6). The required infall velocities are low over the inner few hundred parsecs, only a few $\mathrm{km} \mathrm{s}^{-1}$, and the $\mathrm{H}$ I kinematics are compatible with our 
limits on the streaming velocities $\left(v_{\text {rad }}\right.$ is roughly a few tens of $\left.\mathrm{km} \mathrm{s}^{-1} ; \S 5.3\right)$. We may also conclude that the inferred $\mathrm{H} \mathrm{I}$ mass of the central galactic disks of Seyfert galaxies is sufficient to power the central engine over its lifetime.

Bringing the gas to the central engine poses a more serious problem. The main obstacle is removing sufficient angular momentum to bring gas down to the inner parsec or so, where viscous dissipation takes over (e.g., as reviewed by Phinney 1994). Stellar bars provide gravitational torque and may promote cloud-cloud collisions, funneling gas down at least as far as an inner orbital resonance. Shlosman, Frank, \& Begelman (1989) proposed that mass accumulation at resonances can trigger the formation of nested bars. In principle, bars might nest down to arbitrarily small scales, carrying gas down to correspondingly small volumes.

A basic test of this scheme is to compare the frequency of bars among active and nonactive spiral galaxies. Taking advantage of new near-infrared imaging arrays, recent surveys have found that, in general, spiral galaxies are frequently barred $(\sim 70 \%)$, but the incidences of bars among active and nonactive spiral galaxies are comparable (Mulchaey \& Regan 1997). Moreover, the presence or strength of a bar appears to have little or no impact on the strength of the nuclear activity (Ho, Filippenko, \& Sargent 1997). As mass accumulates at the center of a bar, stellar orbits in the bar are perturbed, leading to the destruction of the bar (e.g., Friedli \& Benz 1993; Norman, Sellwood, \& Hasan 1996). This mechanism might explain the absence of bars in a fraction of Seyfert nuclei. Nevertheless, the results of the imaging surveys raise two questions for the barfueling scenario. First, there is growing evidence for black holes in the centers of both active and nonactive galaxies (e.g., Kormendy \& Richstone 1995; Miyoshi et al. 1995; Gallimore et al. 1996a; Eckart \& Genzel 1996). Why are the black holes in barred, nonactive spirals dormant? Second, other than the presence of a bar, what parameter might distinguish the host galaxies of active and nonactive spirals?

Our study suggests that the gas mass of the central galactic disks may be an important criterion for distinguishing active and nonactive spirals. The high detection rate of $\mathrm{H}$ I absorption argues that the central, 100 pc scale disks of Seyfert nuclei are gas rich, with azimuthally averaged surface densities $\Sigma_{\mathrm{H}}($ Seyfert $) \gtrsim 6\left(C_{f}[\mathrm{H} \mathrm{I}] / 0.7\right) M_{\odot} \mathrm{pc}^{-2}$, where $C_{f}[\mathrm{H} \mathrm{I}]$ is the areal covering fraction of $\mathrm{H}$ I over the central galactic disk, normalized to the detection rate. Such high surface densities should be easily detected in $21 \mathrm{~cm}$ emission in normal spirals. In contrast, nonactive spiral galaxies commonly show an $\mathrm{H}$ I depression over the inner few kiloparsecs (e.g., Roberts 1975; van Woerden, van Driel, \& Schwartz 1983; Giovanelli \& Haynes 1988), with column densities dropping below detections limits of $\sim 1 M_{\odot} \mathrm{pc}^{-2}$.

It is difficult to address this issue further, because there has been no systematic study of central H I depressions in spiral galaxies. The reason is that, except in the dozen or so nearest galaxies, current aperture-synthesis telescopes are generally not sensitive to $\mathrm{H}$ I emission at subkiloparsec resolution. To illustrate, we attempted to generate a comparison sample based on published $\mathrm{H}$ I maps. The selection criteria were (1) Hubble type earlier than $\mathrm{Sb}$, since most Seyfert galaxies reside in early type spiral hosts; and (2) linear resolution better than $1 \mathrm{kpc}$, in order to reduce confusion with the outer disk. We chose galaxies from the
Revised Shapley-Ames catalog (Sandage \& Tammann 1981) and referenced them against the Martin (1998) bibliographic index of $21 \mathrm{~cm}$ maps of galaxies. This selection process picked out 18 candidates for the comparison sample, but all but two of the candidates were either edgeon, active (Seyfert, starburst, or LINER), or strongly interacting with a close companion. This result reflects an observational bias toward the unusual but precludes an meaningful comparison between active and nonactive galaxies.

Nevertheless, we emphasize that feeding the AGN may require not only a stellar bar but also an excess of gas mass in the inner kiloparsec as a source of fuel. Numerical studies of gasdynamics find that stellar bars remove a steady fraction $f_{\text {bar }}$ of the local gas mass per rotation; $0.01 \lesssim f_{\text {bar }} \lesssim 0.1$ (Athanassoula 1992, 1994; Wada \& Habe 1992; Friedli \& Benz 1993; Heller \& Shlosman 1994). The gas mass required to fuel an AGN through bar feeding is

$$
M=1.01 \times 10^{7} L_{44}\left(\frac{\epsilon}{0.1}\right)^{-1}\left(\frac{f_{\text {bar }}}{0.1}\right)^{-1} \Omega_{100}^{-1} M_{\odot},
$$

where $L_{44}$ is the luminosity of the AGN in units $10^{44} \mathrm{ergs}$ $\mathrm{s}^{-1}$ and $\Omega_{100}$ is the bar pattern speed in units $100 \mathrm{~km} \mathrm{~s}^{-1}$ $\mathrm{kpc}^{-1}$. Accepting the considerable uncertainties in the efficiencies, the observed H I absorption masses in active spirals, which give a lower limit to the total gas mass over the central disk, are comparable to the numerical predictions for bar feeding. Otherwise having a stellar bar and a central, supermassive black hole, nonactive spirals may simply lack a central fuel source to power an AGN or nuclear starburst. Testing this scenario will require new $\mathrm{H} \mathrm{I}$ emission observations of a sample of active and nonactive spiral galaxies, reasonably matched in Hubble type and physical resolution (distance). The prediction is that, over the inner kiloparsec, the mean H I surface density of Seyfert galaxies will exceed the $\mathrm{H}$ I surface density of nonactive spirals.

\section{CONCLUSIONS}

Our main conclusions are as follows.

1. H I $21 \mathrm{~cm}$ absorption in Seyfert and starburst galaxies appears to trace gas in the central disks of the host galaxies. The radial scale of the central absorbing disks is hundreds of parsecs (i.e., less than $1 \mathrm{kpc}$ ). High-velocity $\mathrm{H}$ I absorption, Doppler-shifted away from the systemic velocity of the host galaxy, can usually be explained by normal rotational motion rather than radial streaming (e.g., NGC 2110).

2. The $\mathrm{H}$ I kinematics are compatible with, but do not require, streaming velocities of several tens of $\mathrm{km} \mathrm{s}^{-1}$. The corresponding mass inflow rates can be several $M_{\odot} \mathrm{yr}^{-1}$, generally exceeding the mass transfer rate required to fuel a Seyfert nucleus.

3. NGC 4151 appears to be a special case (Mundell et al. 1995). The kinematics and distribution of the $\mathrm{H}$ I absorption are consistent with a compact, parsec-scale disk oriented perpendicular to the radio jet. In contrast, the $\mathrm{H}$ I properties are not compatible with the larger scale galaxy disk orientation and rotation sense.

4. H I absorption in Seyfert galaxies generally avoids the nucleus but is more commonly seen against an extended radio component. The $21 \mathrm{~cm} \mathrm{H}$ I columns are not correlated with the hydrogen column derived from X-ray spectros- 
copy, even in known hidden Seyfert 1 nuclei. We propose that Seyfert nuclei are commonly free-free absorbed and no $21 \mathrm{~cm}$ continuum from the AGN escapes to the neutral layer of the obscuring medium. Alternatively, bright nuclear continuum sources, such as in Mrk 348, can enhance the spin temperature and suppress $21 \mathrm{~cm}$ line absorption. These suppression mechanisms constrain the size of the obscuring medium to smaller than a few tens of parsecs.

5. We suggest that the central disks of active spiral galaxies may show higher $\mathrm{H}$ I surface densities compared with nonactive spirals. This result may resolve the observational challenges for the bar fueling model of AGNs. That is, to be active a spiral galaxy might require both a central stellar bar and an excess of neutral gas. Testing this prediction will require further studies of $21 \mathrm{~cm}$ emission from matched samples of active and nonactive spirals. It is not clear from this study whether we are witnessing gas en route from the outer galaxy (i.e., the fuel line) or a local reservoir from which the nucleus derives its fuel (i.e., the fuel tank).

We would like to acknowledge helpful conversations with Andrew Baker, Mort Roberts, Andrew Wilson, and Jim Ulvestad regarding clarifications and the conclusions of this work. We thank the staff at the VLA Array Operations Center for technical assistance with the observations and data reduction. J. F. G. received support from the STScI Collaborative Visitor's program and a Jansky Fellowship at NRAO, Charlottesville.

\section{APPENDIX A}

\section{COMMENTS ON THE INDIVIDUAL SOURCES}

\section{A1. DETECTED SOURCES}

Mrk 6 is a Seyfert 1.5 galaxy. H I absorption was originally reported by Heckman, Balick, \& Sullivan (1978) and Baan \& Haschick (1983), whose detections we confirm. We have reported a more detailed follow-up MERLIN observation (Gallimore et al. 1998). The nuclear radio source, marginally resolved by the present observation, collimates into a $1^{\prime \prime}$ ( 360 pc) radio jet (Kukula et al. 1996). H I absorption is detected against the northernmost radio component of the $1^{\prime \prime}$ nuclear jet. The offset absorption appears to arise from a 100 pc-scale arm or dust lane crossing just north of the AGN. The AGN itself may be partially absorbed by the dust lane, which may lend to its classification as a Seyfert 1.5. Otherwise, there are no radio candidates for the AGN. Recent $A S C A$ spectroscopy suggests a high emission measure to the nucleus, so the radio nucleus may be free-free absorbed (Feldmeier et al. 1999).

Mrk 231 is a luminous and morphologically peculiar Seyfert 1 galaxy. H I absorption was first reported by Heckman et al. (1978) and (van der Hulst, Hummel, \& Dickey 1982). Our VLA spectra confirm these earlier detections. The VLBA observations of Carilli et al. (1997) resolve the $\mathrm{H}$ I absorption against a $0.44(\sim 350 \mathrm{pc})$ radio continuum disk surrounding the AGN. The $\mathrm{H}$ I absorption displays a $\sim 220 \mathrm{~km} \mathrm{~s}^{-1}$ velocity gradient along the major axis of the diffuse radio continuum disk, and Carilli et al. (1997) argue that the $\mathrm{H}$ I absorption arises in the atomic component of a molecular disk mapped by millimeter-wave CO emission (Bryant \& Scoville 1996).

NGC 1068 is the prototype of Seyfert 2 nuclei but is also the first Seyfert found to have a Seyfert 1 spectrum in polarized light (Antonucci \& Miller 1985). Baan \& Haschick (1983) originally reported H I absorption seen as a sharply defined trough in the $\mathrm{H}$ I emission profile. Our observations resolve out $\mathrm{H} \mathrm{I}$ emission, and the $\mathrm{H} \mathrm{I}$ absorption is spatially resolved. The main results were originally presented in Gallimore et al. (1994). The bulk of the absorption traces the inner disk of the host galaxy, seen against the southwestern radio structure. There is kinematic evidence that the gas is responding to the kiloparsec scale stellar bar. Closer to the nucleus the absorption lines broaden, and there appear multiple velocity components. The opacity of the absorption-line gas also appears to increase, but toward the nucleus the opacity seems to drop sharply. VLBI studies have failed to detect the nucleus (radio component S1) in $21 \mathrm{~cm}$ radio continuum (Roy et al. 1998). The apparent drop in opacity is probably a resolution effect owing to the lack of a background radio source. There may be a broad absorption profile very near the nucleus, whose kinematics are consistent with the rotating $\mathrm{H}_{2} \mathrm{O}$ maser disk (Gallimore et al. 1996a), but bandpass calibration uncertainties preclude a confident detection in the present observations.

NGC 2110 is an X-ray bright Seyfert 2 galaxy (McClintock et al. 1979). At subarcsecond resolution, the "figure-eight" radio structure of our $21 \mathrm{~cm}$ continuum image resolves into a lazy-S-shaped radio jet (Wilson \& Ulvestad 1983). We detect redshifted $\mathrm{H}$ I absorption offset to the south toward the weaker radio lobe.

NGC 2992 is a narrow line X-ray galaxy (Ward et al. 1980). The radio structure comprises an unresolved nuclear source surrounded by figure-eight loops (Wehrle \& Morris 1988). H I absorption is detected offset from the radio nucleus against one of the extended lobes. The host galaxy is viewed nearly edge-on, and so it seems likely that the H I absorption traces gas in the foreground disk.

NGC 3079 is classified as a LINER (Heckman 1980) with evidence for active star formation (e.g., Condon \& Broderick 1988; Irwin \& Seaquist 1990). The H I absorption was discussed briefly by Gallimore et al. (1994) but in more detail by Baan \& Irwin (1995). MERLIN observations well resolve the rotational trend of the absorption-line gas (Pedlar et al. 1996), which matches the sense of the rotation traced by H I emission (Irwin \& Seaquist 1991). The bulk of the absorption probably arises from the edge-on but normally rotating disk, observed in silhouette against extended, diffuse radio emission associated with star formation (Pedlar et al. 1996). Based on new VLBI observations, Satoh et al. (1998) report that the H I absorption viewed toward the central VLBI radio jet appears to be counterrotating relative to the gas motions in the outer galaxy, but it is unclear whether the H I absorption on VLBI scales traces gas in rotation as opposed to, say, nuclear-driven outflow. The 
apparent complexity of the broad absorption-line profile may reflect the structure of the VLBI jet as much as the kinematics and distribution of the absorbing gas.

NGC 3504 is a barred starburst nucleus originally included on Sérsic's list of peculiar nuclei(Sérsic 1973). The nuclear radio emission comprises a compact, unresolved core surrounded by a $\sim 10^{\prime \prime}$ diffuse halo (e.g., Saikia et al. 1994). The halo probably traces synchrotron emission from a 12" diameter starburst ring, revealed in near-infrared color maps (Elmegreen et al. 1997). Dickey (1986) reported a tentative detection of $\mathrm{H}$ I absorption. We clearly detect an absorption line toward the unresolved radio nucleus of this galaxy.

NGC 4151 is a well-studied Seyfert 1.5 galaxy. H I absorption was originally detected in this experiment and followed-up by MERLIN observations (Mundell et al. 1995). The nuclear radio structure resolves into a $\sim 5^{\prime \prime}(\sim 320 \mathrm{pc})$ radio jet (Pedlar et al. 1993, and references therein). H I absorption is detected only toward the radio component C4 (Mundell et al. 1995), which itself resolves into a double source on VLBI baselines (Harrison et al. 1986). The tight confinement of the $\mathrm{H}$ I absorption to the VLBI portion of the radio jet argues that the absorption arises from near-nuclear gas, perhaps a disk surrounding the central engine, at tens of parsecs from the central engine (Mundell et al. 1995).

NGC 5506 is an X-ray bright Seyfert 2 galaxy (Wilson et al. 1976; Rubin 1978). H I absorption was originally reported by Thuan \& Wadiak (1982). The radio emission is dominated by a compact radio source, unresolved at $\sim 0$ ". 1 resolution (Wehrle $\&$ Morris 1987). A low surface brightness arc extends 0".5 north from the nucleus (Unger et al. 1986; Wehrle \& Morris 1987), and faint emission extends $\sim 30^{\prime \prime}$ from the nucleus (Colbert et al. 1996). The host galaxy is viewed nearly edge-on, and it seems likely that most of the absorption arises in foreground disk gas.

\section{A2. NONDETECTIONS}

Mrk 3 is a Seyfert 2 nucleus harboring a hidden BLR (Miller \& Goodrich 1990). The radio structure is linear, resolving into a $2^{\prime \prime}(\sim 600$ pc) jet (Kukula et al. 1993). Surprisingly, we do not detect H I absorption in this hidden Seyfert 1 . On the other hand, there are no flat spectrum components that clearly mark the location of the central engine (Kukula et al. 1993). It may be that the nuclear radio source is free-free suppressed or radio silent, so there is no background radio source to illuminate the material obscuring the central engine.

Mrk 348 (NGC 262) is a Seyfert 2 galaxy that is unusual in two respects. First, it has a very large H i disk grown perhaps by a tidal encounter with a neighboring spiral (Heckman et al. 1982; Simkin et al. 1987). Second, there is evidence for a hidden BLR in this source (e.g., Miller \& Goodrich 1990. Although the nucleus hosts a very bright subarcsecond radio triple (e.g., Neff \& de Bruyn 1983), we detect no H I absorption in this experiment. Halkides, Ulvestad, \& Roy (1997) report evidence for free-free absorption below $6 \mathrm{~cm}$ wavelength, but it is unclear which components of the radio triple might be absorbed. (This result is consistent with the spectral decomposition of Neff \& de Bruyn 1983). The lack of $\mathrm{H}$ I absorption from the medium obscuring the BLR might again be ascribed to free-free suppression of the nuclear radio continuum source at $21 \mathrm{~cm}$ wavelength.

Mrk $668(O Q+208)$ has the optical spectrum of a Seyfert 1 but, given its radio power, may be more appropriately classified as a broad-line radio galaxy (e.g., Stanghellini et al. 1997 and references therein). Mrk 668 is also notable for being one of the nearest gigahertz-peaked spectrum radio sources (O'Dea, Baum, \& Stanghellini 1991). VLBI observations resolve the compact radio source into a 7 mas ( $\sim 5 \mathrm{pc}$ ) double (Stanghellini et al. 1997).

NGC 2639 is a LINER hosting an $\mathrm{H}_{2} \mathrm{O}$ megamaser (Wilson, Braatz, \& Henkel 1995). As may also be the case for the megamaser sources NGC 1068 and NGC 4258, free-free absorption may suppress $21 \mathrm{~cm}$ continuum within the maser disk (Gallimore et al. 1997; Herrnstein et al. 1998). This may explain the lack of $\mathrm{H}$ I absorption corresponding to the $\mathrm{H}_{2} \mathrm{O}$ maser. Alternatively, there may be very little atomic gas associated with the $\mathrm{H}_{2} \mathrm{O}$ masers, but that seems unlikely in the photodissociative environment of an AGN. The radio emission resolves into a 1".4 linear structure (Ulvestad \& Wilson 1989), but, mainly because no spectral index information is available, there is presently no radio candidate for the AGN.

\section{REFERENCES}

Antonucci, R. 1993, ARA\&A, 31, 473

Antonucci, R. R. J., \& Miller, J. S. 1985, ApJ, 297, 621

Athanassoula, E. 1992, MNRAS, 259, 345 1994 , in Mass Transfer Induced Activity in Galaxies, ed. I.

Shlosman (Cambridge: Cambridge Univ. Press), 143

Awaki, H., Koyama, K., Inoue, H., \& Halpern, J. P. 1991, PASJ, 43, 195

Baan, W. A., \& Haschick, A. D. 1983, AJ, 88, 1088

Baan, W. A., \& Irwin, J. A. 1995, ApJ, 446, 602

Baars, J. W. M., Genzel, R., Pauliny-Toth, I. I. K., \& Witzel, A. 1977, A\&A, 619,99

Bahcall, J. N., \& Ekers, R. D. 1969, ApJ, 157, 1055

Begeman, K. G. 1987, Ph.D. thesis, Univ. Groningen

Bosma, A. 1978, Ph.D. thesis, Univ. Groningen

Braun, R. 1997, ApJ, 484, 637

Brinks, E., Skillman, E. D., Terlevich, R. J., \& Terlevich, E. 1997, Ap\&SS, 248, 23

Broeils, A. H., \& van Woerden, H. 1994, A\&AS, 107, 129

Bryant, P. M., \& Scoville, N. Z. 1996, ApJ, 457, 678

Carilli, C. L. 1991, VLA Test Memo. 158 (Socorro: NRAO)

Carilli, C. L., Wrobel, J. M., \& Ulvestad, J. S. 1997, AJ, 115, 928

Carral, P., Turner, J. L., \& Ho, P. T. P. 1990, ApJ, 362, 434

Clements, E. D. 1981, MNRAS, 197, 829

Colbert, E. J. M., Baum, S. A., Gallimore, J. F., O’Dea, C. P., \&

Christensen, J. A. 1996, ApJ, 467, 551
Colbert, E. J. M., Baum, S. A., O'Dea, C. P., \& Veilleux, S. 1998, ApJ, 496, 786

Cole, G. H. J., Pedlar, A., Mundell, C. G., Gallimore, J. F., \& Holloway, A. J. 1998, MNRAS, 301, 782

Colina, L., Fricke, K. J., Kollatschny, W., \& Perryman, M. A. C. 1987, A\&A, 178, 51

Condon, J. J., \& Broderick, J. J. 1988, AJ, 96, 30

Cowie, L. L. 1981, ApJ, 245, 66

de Vaucouleurs, G., de Vaucouleurs, A., Corwin, H. G., Buta, R. J., Paturel, G., \& Fouqué, P. 1991, Third Reference Catalogue of Bright Galaxies (Berlin: Springer)

Dickey, J. M. 1986, ApJ, 300, 190

Dickey, J. M., Terzian, Y., \& Salpeter, E. E. 1978, ApJS, 36, 77

Eckart, A., \& Genzel, R. 1996, Nature, 383, 415

Elmegreen, D. M., Chromey, F. R., Santos, M., \& Marshall, D. 1997, AJ, 114,1850

Elvis, M., Fassnacht, C., Wilson, A. S., \& Briel, U. 1990, ApJ, 361, 459

Feldmeier, J., Brandt, W. N., Elvis, M., Fabian, A. C., Iwasawa, K., \& Mathur, S. 1999, ApJ, 510, 167

Ferland, G. J., \& Osterbrock, D. E. 1986, ApJ, 300, 658

Field, G. B. 1959a, ApJ, 129, 525 1959b, ApJ, 129, 536 $1959 \mathrm{c}$, ApJ, 129, 551

Friedli, D., \& Benz, W. 1993, A\&A, 268, 65 
Gallimore, J. F., Baum, S. A., \& O'Dea, C. P. 1996, ApJ, 464, 198 1997, Nature, 388, 852

Gallimore, J. F., Baum, S. A., O’Dea, C. P., Brinks, E., \& Pedlar, A. 1994, ApJ, 422, L13

1996a, ApJ, 462, 740

Gallimore, J. F., Baum, S. A., O’Dea, C. P., \& Pedlar, A. 1996b, ApJ, 458, 136

Gallimore, J. F., Holloway, A. J., Pedlar, A., \& Mundell, C. G. 1998, A\&A, 333,13

Genzel, R., Watson, D. M., Crawford, M. K., \& Townes, C. H. 1985, ApJ, 297,766

George, I. M., Turner, T. J., Netzer, H., Nandra, K., Mushotzky, R. F., \& Yaqoob, T. 1998, ApJS, 114, 73

Giovanelli, R., \& Haynes, M. P. 1988, in Galactic and Extragalactic Radio Astronomy, ed. G. L. Verschuur \& K. I. Kellermann (Berlin: Springer), 522

Halkides, D., Ulvestad, J., \& Roy, A. 1997, BAAS, 191, 104.04

Harrison, B., Pedlar, A., Unger, S. W., Burgess, P., Graham, D. A., \&

Preuss, E. 1986, MNRAS, 218, 775

Heckman, T. M. 1980, A\&A, 87, 152

Heckman, T. M., Balick, B., \& Sullivan, W. T., III. 1978, ApJ, 224, 745

Heckman, T. M., Sancisi, R., Balick, B., \& Sullivan, W. T., III. 1982, MNRAS, 199, 425

Helfer, T. T., \& Blitz, L. 1995, ApJ, 450, 90 1997, ApJ, 478, 162

Helfer, T. T., Thornley, M. D., Regan, M. W., Sheth, K., Vogel, S. N. Wong, T., Blitz, L., \& Bock, D. 1998, BAAS, 192, 7301

Heller, C. H., \& Shlosman, I. 1994, ApJ, 424, 84

Herrnstein, J. R., Greenhill, L. J., Moran, J. M., Diamond, P. J., Inoue, M.,

Nakai, N., \& Miyoshi, M. 1998, ApJ, 497, 89

Ho, L. C., Filippenko, A. V., \& Sargent, W. L. W. 1997, ApJ, 487, 591

Huchra, J., Gellar, M. J., de Lapparent, V., \& Corwin, H. G. J. 1990, ApJS, 72,433

Irwin, J. A., \& Seaquist, E. R. 1990, ApJ, 353, 469

. 1991, ApJ, 371, 111

Jackson, J. M., Heyer, M. H., Paglione, T. A. D., \& Bolatto, A. D. 1996, ApJ, 456, 91

Keel, W. C. 1980, AJ, 85, 198

Kenney, J. D. P., Carlstrom, J. E., \& Young, J. S. 1993, ApJ, 418, 687

Kennicutt, R. C. 1989, ApJ, 344, 685

. 1990, ApJ, 498, 541

Kormendy, J., \& Richstone, D. 1995, ARA\&A, 33, 581

Koski, A. 1978, ApJ, 223, 56

Kriss, G. A., et al. 1992, ApJ, 392, 485

Krolik, J. H., \& Lepp, S. 1989, ApJ, 347, 179

Kukula, M. J., Ghosh, T., Pedlar, A., \& Schilizzi, R. T. 1999, ApJ, 518, 117

Kukula, M. J., Ghosh, T., Pedlar, A., Schillizzi, R. T., Miley, G. K., de Bruyn, A. G., \& Saikia, D. J. 1993, MNRAS, 264, 893

Kukula, M. J., Holloway, A. J., Pedlar, A., Meaburn, J., Lopez, J. A., Axon, D. J., Schilizzi, R. T., \& Baum, S. A. 1996, MNRAS, 280, 1283

Lawrence, A. 1987, PASP, 99, 309

1991, MNRAS, 252, 586

Lilie, P. 1994, VLA Test Memo. 190 (Socorro: NRAO)

Liszt, H. S., van der Hulst, J. M., Burton, W. B., \& Ondrechen, M. P. 1983, A\&A, 126, 341

Maiolino, R., Stanga, R., Salvati, M., \& Rodriguez Espinosa, J. M. 1994, A\&A, 290, 40

Malkan, M. A., Gorjian, V., \& Tam, R. 1998, ApJS, 117, 25

Maloney, P. R., Hollenbach, D. J., \& Tielens, A. G. G. M. 1996, ApJ, 466, 561

Martin, M. C. 1998, A\&AS, 131, 73

Matt, G., et al. 1997, A\&A, 325, L13

McClintock, J. E., Remillard, R. A., Canizares, C. R., Veron, P., \& van Paradijs, J. 1979, ApJ, 233, 809

Meaburn, J., Whitehead, M. J., \& Pedlar, A. 1989, MNRAS, 241, 1P

Mezger, P. G., \& Henderson, A. P. 1967, ApJ, 147, 471

Miller, J. S., \& Goodrich, R. W. 1990, ApJ, 355, 456

Miyoshi, M., Moran, J., Herrnstein, J., Greenhill, L., Nakai, N., Diamond, P., \& Inoue, M. 1995, Nature, 373, 127

Moran, E. C., Halpern, J. C., Bothun, G. D., \& Becker, R. H. 1992, AJ, 104, 990

Morrison, R., \& McCammon, D. 1983, ApJ, 270, 199

Mulchaey, J. S., Colbert, E., Wilson, A. S., Mushotzky, R. F., \& Weaver, K. A. 1993, ApJ, 414, 144

Mulchaey, J.S., Mushotzky, R. F., \& Weaver, K. A. 1992, ApJ, 390, L69

Mulchaey, J. S., \& Regan, M. W. 1997, ApJ, 482, L135

Mundell, C. G., Holloway, A. J., Pedlar, A., Meaburn, J., Kukula, M. J., \& Axon, D. J. 1995, MNRAS, 275, 67

Mushotzky, R. F., Boldt, E. A., Holt, S. S., Serlemitsos, P. J., Swank, J. H., Rothschild, R. H., \& Pravdo, S. H. 1978, ApJ, 226, L65

Nagar, N. M., Wilson, A. S., Mulchaey, J. S., \& Gallimore, J. F. 1999, ApJS, 120, 209

Neff, S. G., \& de Bruyn, A. G. 1983, A\&A, 128, 318

Nelson, C. H., \& Whittle, M. 1995, ApJS, 99, 67

Neufeld, D. A., Maloney, P. R., \& Conger, S. 1994, ApJ, 436, L127

Norman, C. A., Sellwood, J. A., \& Hasan, H. 1996, ApJ, 462, 114

O'Dea, C. P., Baum, S. A., \& Gallimore, J. F. 1994, ApJ, 436, 669
O’Dea, C. P., Baum, S. A., \& Stanghellini, C. 1991, ApJ, 380, 66

Payne, H. E., Salpeter, E. E., \& Terzian, Y. 1982, ApJS, 48, 199 1983, ApJ, 272, 540

Pedlar, A., Fernandez, B., Hamilton, N. G., Redman, M. P., \& Dewdney, P. E. 1998, MNRAS, 300, 1071

Pedlar, A., Howley, P., Axon, D. J., \& Unger, S. W. 1992, MNRAS, 259, 369

Pedlar, A., Kukula, M., Longley, D. P. T., Muxlow, T. W. B., Axon, D. J., Baum, S., O'Dea, C. P., \& Unger, S. W. 1993, MNRAS, 263, 471

Pedlar, A., Mundell, C. G., Gallimore, J. F., Baum, S. A., \& O'Dea, C. P. 1996, Vistas Astron., 40, 91

Peterson, B. M. 1997, An Introduction to Active Galactic Nuclei (Cambridge: Cambridge Univ. Press)

Phinney, E. S. 1994, in Mass Transfer Induced Activity in Galaxies, ed. I. Shlosman (Cambridge: Cambridge Univ. Press), 1

Press, W. H., Teukolsky, S. A., Vetterling, W. T., \& Flannery, B. P. 1992 Numerical Recipes (2d ed.; Cambridge: Cambridge Univ. Press)

Read, A. M., Ponman, T. J., \& Strickland, D. K. 1997, MNRAS, 286, 626

Rees, M. J. 1984, ARA\&A, 22, 471

Rigopoulou, D., Lawrence, A., White, G. J., Rowan-Robinson, M., \& Church, S. E. 1996, A\&A, 305, 747

Roberts, M. S. 1975, in Galaxies and the Universe, ed. A. Sandage, M. Sandage, \& J. Kristian (Chicago: Univ. Chicago Press), 309

Roberts, W. W. J., Huntley, J. M., \& van Albada, G. D. 1979, ApJ, 233, 67

Roy, A. L., Colbert, E. J. M., Wilson, A. S., \& Ulvestad, J. S. 1998, ApJ, 504, 147

Rubin, V. C. 1978, ApJ, 224, L55

Saikia, D. J., Pedlar, A., Unger, S. W., \& Axon, D. J. 1994, MNRAS, 270, 46

Sandage, A., \& Tammann, G. A. 1981, Revised Shapley-Ames Catalog of Bright Galaxies (Washington: Carnegie Instit. Washington)

Sanders, D. B., Phinney, E. S., Neugebauer, G., Soifer, B. T., \& Matthews, K. 1989, ApJ, 347, 29

Sanders, R. H., \& Tubbs, A. D. 1980, ApJ, 235, 803

Satoh, S., Inoue, M., \& Nakai, N. 1998, in ASP COnf. Ser. 144, Radio Emission from Galactic and Extragalactic Compact Sources, IAU Colloq. 164, ed. J. A. Zensus, G. B. Taylor, \& J. M. Wrobel (San Francisco: ASP), 219

Schmitt, H. R., Kinney, A. L., Storchi-Bergmann, T., \& Antonucci, R. 1997, ApJ, 422, 623

Schwarz, U. J., Ekers, R. D., \& Goss, W. M. 1982, A\&A, 110, 100

Sérsic, J. L. 1973, PASP, 85, 103

Shlosman, I., Frank, J., \& Begelman, M. C. 1989, Nature, 338, 45

Shostak, S., \& van der Kruit, P. C. 1984, A\&A, 132, 20

Simcoe, R., McLeod, K. K., Schachter, J., \& Elvis, M. 1997, ApJ, 489, 615

Simkin, S. M., van Gorkom, J., Hibbard, J., \& Su, H.-J. 1987, Science, 235 1367

Smith, D., \& Done, C. 1996, MNRAS, 280, 355

Stanghellini, C., Bondi, M., Dallacasa, D., O’Dea, C. P., Baum, S. A., Fanti, R., \& Fanti, C. 1997, A\&A, 318, 376

Thuan, T. X., \& Wadiak, E. J. 1982, ApJ, 252, 125

Toomre, A. 1964, ApJ, 139, 1217

Tran, H. D., Miller, J. S., \& Kay, L. E. 1992, ApJ, 397, 457

Turner, T. J., George, I. M., Nandra, K., \& Mushotzky, R. F. 1997, ApJS, 113,23

Ulvestad, J. S., Roy, A. L., Colbert, E. J. M., \& Wilson, A. S. 1998, ApJ, 496, 196

Ulvestad, J. S., \& Wilson, A. S. 1984, ApJ, 285, 439

1989, ApJ, 343, 659

Ulvestad, J. S., Wrobel, J. M., Roy, A. L., Wilson, A. S., Falcke, H., \& Kritchbaum, T. P. 1999, ApJ, 517, L81

Unger, S. W., Pedlar, A., Booler, R. V., \& Harrison, B. A. 1986, MNRAS 219,387

Unger, S. W., Pedlar, A., Neff, S. G., \& de Bruyn, A. G. 1984, MNRAS, 209, $15 \mathrm{p}$

van der Hulst, J. M., Hummel, E., \& Dickey, J. M. 1982, ApJ, 261, L59

van Driel, W. 1987, Ph.D. thesis, Univ. Groningen

van Gorkom, J. H., Knapp, G. R., Ekers, R. D., Ekers, D. D., Laing, R. A., \& Polk, K. S. 1989, AJ, 97, 708

van Woerden, H., van Driel, W., \& Schwartz, U. J. 1983, in Internal Kinematics and Dynamics of Galaxies, ed. E. Athanassoula (Dordrecht: Reidel), 99

Wada, K., \& Habe, A. 1992, MNRAS, 258, 82

Wang, T., Brinkmann, W., \& Bergeron, J. 1996, A\&A, 309, 81

Ward, M., Penston, M. V., Blades, J. C., \& Turtle, A. J. 1980, MNRAS, 193, 563

Ward, M. J. 1996, Vistas Astron., 40, 233

Wehrle, A. E., \& Morris, M. 1987, ApJ, 313, L43

1988, AJ, 95, 1689

Whittle, M. 1992, ApJS, 79, 49

Wilson, A. 1991, in The Interpretation of Modern Synthesis Observations of Spiral Galaxies, ed. N. Duric \& P. C. Crane (San Francisco: ASP), 227

Wilson, A. S., \& Baldwin, J. A. 1985, ApJ, 289, 124

Wilson, A. S., Braatz, J. A., \& Henkel, C. 1995, ApJ, 455, L127

Wilson, A. S., Penston, M. V., Fosbury, R. A. E., \& Boksenberg, A. 1976, MNRAS, 177,673

Wilson, A. S., \& Ulvestad, J. S. 1983, ApJ, 275, 8 